\title{
MEASURING INTER-PARTICLE FORCES AT AN INTERFACE WITH OPTICAL TWEEZERS AND A LONG WORKING-DISTANCE OBJECTIVE LENS
}

\author{
by
}

Daniel R. McAdams

B.S. in Physics, Pennsylvania State University, 2006

\author{
Submitted to the Graduate Faculty of \\ the Swanson School of Engineering in partial fulfillment \\ of the requirements for the degree of \\ Master of Science
}

University of Pittsburgh

2009 


\section{UNIVERSITY OF PITTSBURGH SWANSON SCHOOL OF ENGINEERING}

This thesis was presented

by

Daniel R. McAdams

It was defended on

December 9, 2008

and approved by

Daniel G. Cole, Ph. D., Professor

Sachin Valenkar, Ph. D., Professor

William Clark, Ph. D., Professor

Thesis Advisor: Daniel G. Cole, Ph. D., Professor 


\title{
MEASURING INTER-PARTICLE FORCES AT AN INTERFACE WITH OPTICAL TWEEZERS AND A LONG WORKING-DISTANCE OBJECTIVE LENS
}

Daniel R. McAdams, M.S.

University of Pittsburgh, 2009

\begin{abstract}
An optical tweezer set up is used to measure the forces between polystyrene microspheres on the interface between mineral oil and water. The trap is controlled through Matlab Simulink and dSpace ControlDesk. The forces were found to follow a combination of Coulomb's law and dipole-dipole interaction, but for much less charge than is specified by the manufacturer to be on the surface of the microspheres. There is also a value given for the effective viscosity of the interface. A discussion on the design and motivation for using a Bessel beam trap can be found in the appendix.
\end{abstract}




\section{TABLE OF CONTENTS}

1.0 INTRODUCTION . . . . . . . . . . . . . . . . . . . . . 1

1.1 Background and Motivation . . . . . . . . . . . . . . 5

1.1.1 Colloidal Experiments . . . . . . . . . . . . . . . . . 7

2.0 OPTICAL TRAPPING THEORY AND SET UP . . . . . . . . . . 9

2.1 Optical Tweezer Theory . . . . . . . . . . . . . . . . . 9

2.1.1 Mie Scattering . . . . . . . . . . . . . . . 12

2.1.2 Optical Trap set-up . . . . . . . . . . . . . . 14

2.2 System Characterization . . . . . . . . . . . . . . . . . . 22

2.3 Actuation by Fast-Steering Mirror . . . . . . . . . . . . . . . . . . 23

2.4 Sensing . . . . . . . . . . . . . . . . . . . . . 23

2.4.1 Quadrant Photo Diode (QPD) . . . . . . . . . . . 24

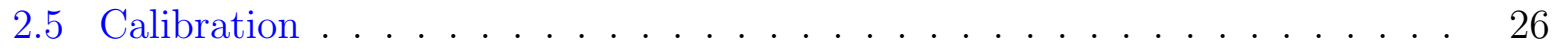

2.5.1 FSM volts to Trap displacement . . . . . . . . . . . . 26

2.5.2 Displacement of the bead from the trap . . . . . . . . . . . . . 29

2.5.3 Trap Stiffness . . . . . . . . . . . . . . . . . . . . 38

2.5.3.1 Equipartition Method ... . . . . . . . . . 39

2.5.3.2 Frequency Spectrum Method . . . . . . . . . . . . . 41

3.0 COLLOIDAL INTERACTION ENERGIES AT AN INTERFACE . . . 43

3.1 Van der Waals Forces . . . . . . . . . . . . . . . . . . . . . . . . . . 44

3.2 Capillary forces and Surface Tension . . . . . . . . . . . . . . . 45

3.3 Electrostatic Forces . . . . . . . . . . . . . . . . . . . . . 46

4.0 EXPERIMENT . . . . . . . . . . . . . . . . . . . 54 


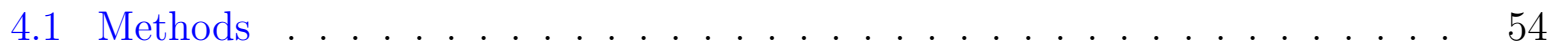

5.0 RESULTS AND DISCUSSION . . . . . . . . . . . . . . . . 57

5.1 Assumptions . . . . . . . . . . . . . . . . . . . . . 59

5.2 Gaussian Trap Pair Potential Measurements . . . . . . . . . . . . . . 59

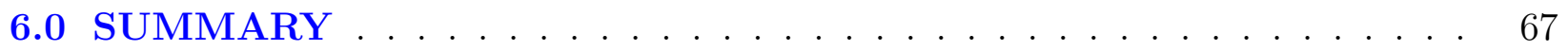

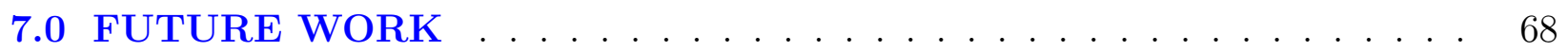

7.1 Bessel Beams . . . . . . . . . . . . . . . . . . . . . . . . . . . 69

7.1 .1 Choosing an Axicon . . . . . . . . . . . . . . . . . 77

7.1.2 Preliminary Results and Observations . . . . . . . . . . . . . 80

7.2 Bessel Beam Trap Pair Potential Measurements . . . . . . . . . . . . . . . . 80

APPENDIX. WHOLE PROCEDURE . . . . . . . . . . . . . . . 82






\section{LIST OF TABLES}

1 Specifications of CCD camera. . . . . . . . . . . . . . . 20

2 Conversions in Force Calculation. . . . . . . . . . . . . . . . . . . . . . . . 29

$3 \quad$ FSM volts per pixel of beam movement across the screen for the Gaussian trap in bulk water and on the interface. . . . . . . . . . . . . . . . . 29

4 The table shows the average dynamic range of QPD response to scanning the laser across a fixed bead. This shows that the ratio between trap power and low power is much more important than the combination of media. . . . . . . 33

5 Densities of Materials in Experiment in grams per cubic centimeter . . . . . 45

6 Dielectric Constants of Materials in Experiment. . . . . . . . . . . . 51

7 Conversions in Force Calculation. . . . . . . . . . . . . . . . . . . . . 59

8 Microsphere Information. . . . . . . . . . . . . . . . . 64 


\section{LIST OF FIGURES}

1 Light interacts with a colloidal particle in such a way to cause a restoring force toward the regions of highest intensity gradient $[16] \ldots \ldots$. . . . . . . . 2

2 Model of the well used in the experiment (5 mm in diameter) . . . . . . . . 4

3 The higher the numerical aperture, the greater the angle of rays collected by the lens. . . . . . . . . . . . . . . . . . . . 6

4 The different refractive indices that the beam encounters as it makes its way through the sample. . . . . . . . . . . . . . . . . 11

5 If objective lens has a high enough NA, the bead always feels a force restoring it to the focus of the beam. a.) Shows that a bead beyond the focus of the beam, is pulled backward. b.) Shows that a bead centered behind the focus is pushed forward. c.) Shows that a bead that is to the right of the focus, is pushed to the left $[45] \ldots \ldots \ldots \ldots \ldots$

6 Optical Tweezer Setup with Gaussian beam. . . . . . . . . . . . . 16

7 Schematic of the variables used to calculate the force between the beads. . . . 18

8 Free body diagram of forces on bead. The equation of motion can be derived from this. . . . . . . . . . . . . . . . . . . . . 19

9 Sensing Portion of Set-up. . . . . . . . . . . . . . . . . . 21

10 Two of the most important signals coming from the qpd are the XDIFF and YDIFF signals. The XDIFF signal is the difference in current between the right and left half of the qpd. The YDIFF signal is the difference in current between the top and bottom half of the qpd. . . . . . . . . . . . 
11 Schematic representation of how a change in relative bead/beam position does not move the spot on the QPD, but does change the position of the intensity maximum, allowing for displacement measurement. The spot on the QPD does not move because the specimen and QPD are at conjugate Fourier planes and the aperture diaphragm cuts off light that would be projected to a different

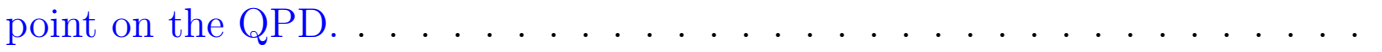

12 The middle row shows the same thing as the previous figure, but the third row shows the effect of putting the QPD somewhere besides the back focal plane $(\mathrm{BFP})$ of the condenser. . . . . . . . . . . . . . . . .

13 Here is an example of an FSM volts to nanometer calibration. The number of pixels in the image between the two extremes (a 6 volt span) in FSM voltage is found. This is compared to the number of nanometers per pixel, found by measuring the known diameter of the image of the microsphere. . . . . . . .

14 This is the QPD voltage from X channel of a bead dried to a coverslip and covered in water. The trap was rastered across the face of the bead. . . . . .

15 This is the QPD voltage from $\mathrm{Y}$ channel of a bead dried to a coverslip and covered in water. The trap was rastered across the face of the bead. . . . . .

16 The QPD voltage response is only linear in the central region of the bead. All measurements must take place with this small displacement from the bead

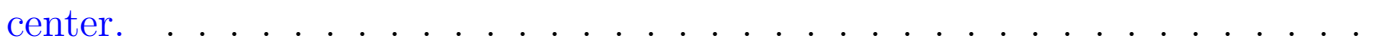

17 Dry beads onto two cover slips. Make one slide with water and one with oil to test the response of the QPD as the beam is scanned across the bead and diffracted. Treating the bead like a slab of polystyrene, ray goes through two refractions. Say that $\theta_{1}=15$ degrees. Both cases give the same resulting angles, but because $\theta_{4}$ is greater in the water case, the displacement of the beam is further from the entrance point than in the oil case. . . . . . . . . . . . . 
18 Dry beads onto two slides. Make one slide with water and one with oil to test the response of the QPD as the beam is scanned across the bead and diffracted. Treating the bead like a slab of polystyrene, ray goes through two refractions. Say that $\theta_{1}=15$ degrees. Both cases give the same resulting angles, but because $\theta_{4}$ is greater in the water case, the displacement of the beam is further from the entrance point than in the oil case. . . . . . . . . . . . . . . 35

19 System with four AA's to make clean measurements. . . . . . . . . . . . 36

20 FSM to QPD transfer function (in linear region) for $\mathrm{y}$ to $\mathrm{y}$ and $\mathrm{y}$ to $\mathrm{x}$ (cross channel). The trailing off of the data around $400 \mathrm{~Hz}$, is due to the $500 \mathrm{~Hz}$ cutoff of the AA filter. . . . . . . . . . . . . . . . . .

21 FSM to QPD transfer function (in linear region) for $\mathrm{x}$ to $\mathrm{x}$ and $\mathrm{x}$ to $\mathrm{y}$ (cross channel). The trailing off of the data around $400 \mathrm{~Hz}$, is due to the $500 \mathrm{~Hz}$ cutoff of the AA filter. . . . . . . . . . . . . . . . .

22 Coherence of FSM to QPD for $\mathrm{y}$ to $\mathrm{y}$ and $\mathrm{y}$ to $\mathrm{x}$. The coherence is near one for all pertinent frequencies in the two corresponding channels (FSMy to QPDy and FSMx to QPDx). The cross channels are not as important, but they are also around one for most of the spectrum. . . . . . . . . . . . .

23 Coherence of FSM to QPD for $\mathrm{x}$ to $\mathrm{x}$ and $\mathrm{x}$ to $\mathrm{y}$. The coherence is near one for all pertinent frequencies in the two corresponding channels (FSMy to QPDy and FSMx to QPDx). The cross channels are not as important, but they are also around one for most of the spectrum. . . . . . . . . . . . . . . . 40

24 Random movements of bead on the interface. . . . . . . . . . . . . . . . . 41

25 Power spectrum of equipartition data with fits of 0.5 and $1 \mathrm{~Hz}$. . . . . . . 42

26 Diagram of surface forces acting on bead, independent of other particles. . . . 47

27 Lattice spacing on edge of a lattice. . . . . . . . . . . . . . . . . . . . 49

28 Lattice spacing in center of band. . . . . . . . . . . . . . 50

29 Dipole repulsion acting only through oil. . . . . . . . . . . . . . . . . . 52

30 Sample with bunches of beads after 18 hrs. . . . . . . . . . . . . . . 58

31 Force on bead with respect to distance from each other in direction perpendicular to movement. . . . . . . . . . . . . . . . . . . . 60 
32 Force on bead with respect to distance from each other in direction perpendicular to movement. . . . . . . . . . . . . . . . . .

33 Force on bead with respect to distance from each other in direction parallel to movement with a Coulomb force fit. . . . . . . . . . . . . . . . .

34 Force on bead with respect to distance from each other in direction parallel to movement with Coulomb force fit. . . . . . . . . . . . . . . . .

35 Force on bead with respect to distance from each other in direction parallel to movement with a Dipole- Dipole force fit. . . . . . . . . . . . . . . . .

36 Force on bead with respect to distance from each other in direction parallel to movement with Dipole-Dipole force fit. . . . . . . . . . . . . . .

37 Force on bead with respect to distance from each other in direction parallel to movement with a Coulomb and Dipole-Dipole force combined fit. . . . . . . . 64

38 The total surface charge taken as a point charge at the center of two interacting beads necessary to match the experimental data. . . . . . . . . . . . . . . 65

39 Gaussian beam characteristics. . . . . . . . . . . . . . . . 70

40 Two dimensional plot of Bessel beam along the optical axis. . . . . . . . . . . 71

41 The use of a Bessel beam more accurately determines a force acting on a particle adsorbed to curved interface. This is because the intensity is the same at all points where the bead comes into contact with the interface. . . . . . . 73

42 Optical Tweezer Setup with Axicon. . . . . . . . . . . . . . . . . . 76

43 Variables in propagation matrix. . . . . . . . . . . . . . . . . . 78 


\subsection{INTRODUCTION}

Photons have momentum. When they interact with matter through reflection, refraction and absorption, they transfer momentum to the object. The transfer of momentum results in a force.

The term "optical trap" or "optical tweezer" refers to how a tightly focused laser beam can be used to manipulate a small particle in two dimensions, or confine an object in a three dimensional potential well. This was first demonstrated by Arthur Ashkin in 1970 [4]. Because the trapping potential acts like a spring, optical traps can be used to measure small forces acting on microparticles (see Figure 1).

There are two major methods to measure forces in the piconewton to micronewton range: optical tweezers and atomic force microscopy. The major advantage of the optical tweezer over the other major micromanipulation technique, atomic force microscopy (AFM), is that the object under study does not come into contact with the measurement device. For example the organelles of a cell can be manipulated while the cell is still alive. If an object cannot be trapped directly, it can still be studied with optical tweezers if a suitable microparticle is attached to the object to act as a handle. Sometimes these handles can interact with each other if they have charge or are chemically active with respect to the fluids in which they are suspended. The trap in this thesis experiment can measure down to the femptonewton range. The reason for this will be explained below.

Colloid science is the study of the interaction between small solid particles and a liquid medium. Colloids are used in many industries, and their stability and other properties are integral to a variety of processes. Fundamental questions about the behavior of certain types of colloids under different conditions remain today. New experiments help refine the theory describing colloids in these situations. The forces between colloidal particles are important in 


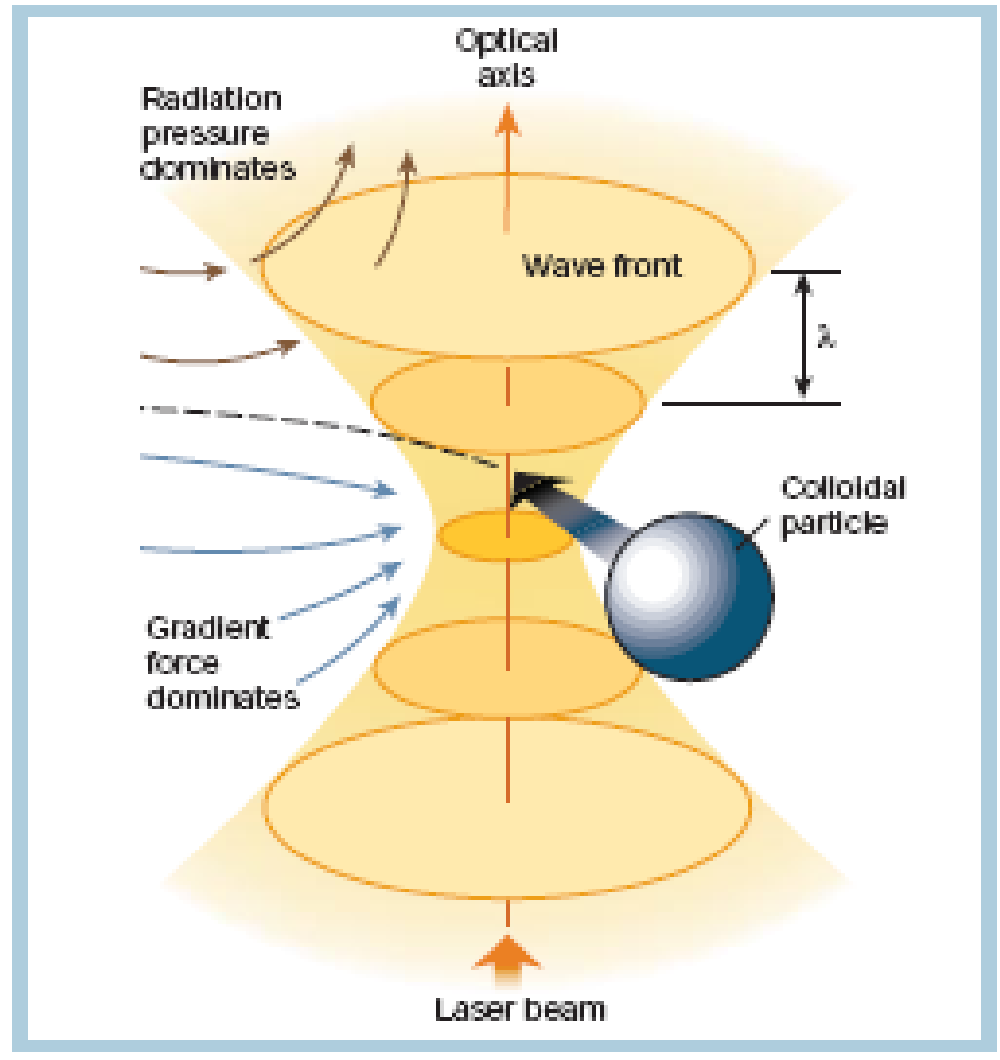

Figure 1: Light interacts with a colloidal particle in such a way to cause a restoring force toward the regions of highest intensity gradient [16]. 
order to improve models and understand some yet-to-be explained phenomena. Information about these forces can be applied to other sciences, e.g. where ordered arrays of particles are needed in photonic crystals. Colloids are also a convenient way to model atomic systems. It is possible to monitor phase transitions on a longer timescale, and in a more controllable manner in colloids than it is in atomic systems [3].

This thesis will discuss the interaction forces that small polystyrene spheres experience when they are adsorbed to the interface between oil and water. The data and the methods with which the data is collected will help answer questions about colloid science. The various forces on the polystyrene beads (van der Waals, capillary, Coulomb and dipoledipole repulsion) will be measured using a traditional Gaussian beam-based trap, and the use of a much less common Bessel beam trap will be discussed as future work. The optical trap is a suitable measurement tool because optical forces are on the order of fempto- to nano-newtons, which is the range of magnitudes for the inter-particle forces being measured.

The forces between the beads will displace them in the traps. The amount of displacement is related to the force by the trap stiffness, $k$. Light scattered by the bead is related to the displacement. Hooke's Law can be used to calculate the force for a given trap stiffness. The stiffness of the trap can be found using the equipartition method as discussed below.

In the experiment, polystyrene beads will be placed on the interface between oil and water, approximately confining them to two dimensions. The force of one bead acting on another will be measured using two optical traps, one for each bead. The interface on which the beads in this experiment lie is formed by placing water and oil in a well (see Figure 2).

Information about the stabilization of colloids by use of small particles can be taken from the experiments discussed in this thesis. This thesis will also add to the body of knowledge available about interfaces and 2D microsphere interaction. The viscosity and various other properties of interfaces are unknown, and normally assumed in the interpretation of many experiments. A value for the effective interfacial viscosity will be given in the Results section. The drag force on a spherical particle at an interface is not known. Because the forces the microspheres exert on each other are highly dependent on the nature of the interfacing fluids and the particles themselves, having accurate knowledge about two of the three substances can provide information about the remaining substance through measurement with an optical 




Figure 2: Model of the well used in the experiment (5 $\mathrm{mm}$ in diameter). 
trap [12]. It may be possible to determine minute variations in surface charge or electrolyte concentration by measuring the forces two microspheres exert on each other.

Normally oil immersion lenses are used for optical trapping experiments because they have a higher numerical aperture (NA). Numerical aperture is a figure describing how wide of an angle of light rays that a lens can collect. A higher NA provides a stronger intensity gradient at the focus as light converges from a greater angle. Unfortunately, oil immersion lenses cannot reach deep into a sample; a long working distance objective can. It may be possible to negate the disadvantages of a long working distance lens (being limited to weaker, 2D trapping because of lower NA), by using a Bessel beam as the trap.

\subsection{BACKGROUND AND MOTIVATION}

There have been several advances and additions of functionality to optical trapping since Ashkin's discovery in 1970 that allow for the measurement of the pair potential/force in this research. These advances were slowly applied to the questions of colloid science.

There are two forces at work in an optical trap, the scattering force and the gradient force (see Figure 1. The scattering force pushes the bead along the optical axis due to photons being reflected, and dominates the gradient force at low NA. The gradient force pulls the bead toward the area of the beam with the highest intensity gradient (the center in a Gaussian beam). The gradient force acts in a radial direction from the focus [5]. The scattering force acting along the beam axis.

The field of optical trapping started out with only lateral trapping capability. In order to stably trap an object in three dimensions, two counter-propagating beams were used. In 1986 Ashkin, Dziedzic, et al. used a high enough NA lens to increase the axial intensity gradient, increasing the restoring force toward the focus [1]. In the ray optics regime $(\lambda \ll$ diameter) only lateral 2D trapping is possible if the convergence angle of the light is less than $\approx 30$ degrees [5]. If the particle is moving toward the focus along the optical axis, it has the gradient force pulling it toward the focus and the scattering force pushing it forward. If the bead is further down the optical axis it is being pulled back toward the focus by the 


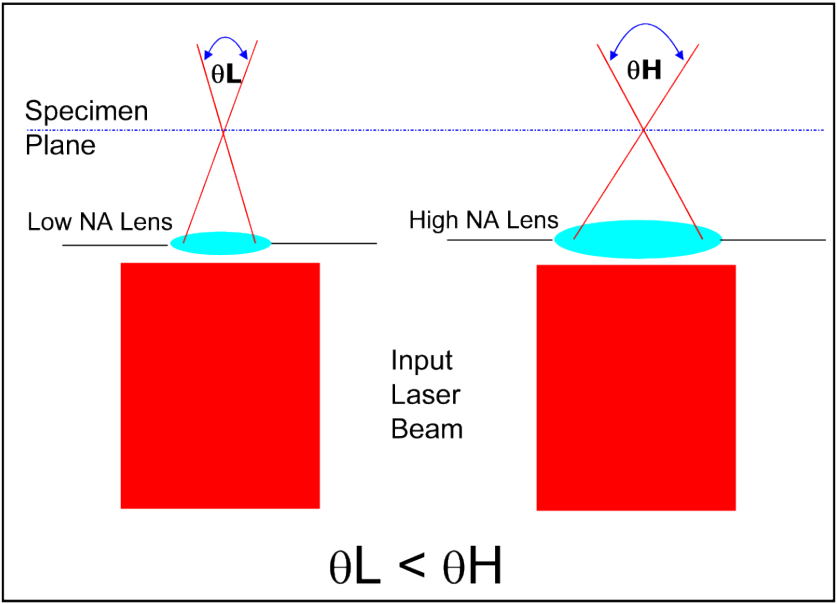

Figure 3: The higher the numerical aperture, the greater the angle of rays collected by the lens.

gradient force and is being pushed away by the scattering force. The force exerted on a particle by a trap is independent of particle radius, $r$, in the ray optics regime, and varies as $r^{3}$ in the Rayleigh regime $(\lambda \gg$ diameter) [5].

At first only image analysis of photographs and video images could be used to determine the position of a trapped particle. In order to move beyond a microscope's resolution limit, a new position detection system needed to be developed. Any external force acting on a microparticle in an optical trap, pushes the particle out of its natural position in the center of the beam. This means that the change in light scattering due to the translation of the particle can be measured and used to accurately determine the amount of displacement and achieve sensing at bandwidths above video sample rates. Then through the knowledge of the stiffness of the spring-like characteristic of the potential created by the laser, the force that caused the particle to be displaced can be found. Ashkin used a photodiode for feedback stabilization of optically levitated particles in 1977 [7]. This method involved trying to keep a trapped particle at the same height above the microscope objective. The signal to the 
photodiode varied with the height of the particle. Two dimensional displacement was first measured with quadrant photodiodes in 1992 [25].

When Ashkin was using a single trap to levitate a microparticle, technically a force measurement was being made. When the particle was motionless, it was known that the force of gravity was balanced with the force of radiation pressure against the bead. The first force measurement made on a force exerted by something under test, i.e. not some force applied outside of the experimental set-up with magnets, gravity, etc. was made in 1989 by equating the Stokes drag on a trapped bacteria with the force necessary to break the bacteria out of the trap, the "escape force" [39]. This is a popular method of calibration.

\subsubsection{Colloidal Experiments}

Ten years after the invention of optical tweezing, Pawel Pieranski reported the first microscopic observation of microspheres arranged in a lattice on an interface in 1980. The interface was between water and air. The particles were found to create a triangular crystal lattice in some cases, and disordered arrays in others [32]. He was able to use a regular microscope objective (instead of a long-working distance objective) by removing the water until there was only a 3 micron thick layer between the coverslip and the air. In the disordered structure he observed longer interaction distances, greater than 10 microns for beads that were only $245 \mathrm{~nm}$ in diameter. The interactions he observed were repulsive.

In 1994, John C. Crocker and David G. Grier were the first to apply laser tweezers to the problem of measuring colloidal interaction potentials. They did not use the lasers as measurement devices though, and simply used them to position the beads in bulk at a specified distance from each other. Then by turning off the lasers, video observations could be made of the beads' reactions to each other at different separations [11]. Force measurements could be deduced from the movement of the beads by use of Stokes drag and diffusion. The beads are spherical and have a known radius. Knowledge of the viscosity of the water can then be used for interaction force determination. They later found evidence

of a long-range attractive potential when the microspheres were located between charged glass walls (within 25 microns for 1 micron diameter beads), similar to many experimental 
set-ups used to make measurements on colloids [13]. The experiment in this thesis does not place particles between charged panes of glass, and therefore should not see evidence of a long-range attractive potential.

The first use of laser tweezers to measure forces between particles on an interface was by Aveyard et al. in 2002. They brought two beads together with a time-shared trap until the repulsive force between the beads forced one out of the trap. In a time-shared trap a single laser is scanned from trap position to trap position, with a repetition rate fast enough that the particles do not have time to wander from the trap due to Brownian motion. By performing this experiment at different powers it is possible to create a bead-to-bead separation distance vs. interaction force graph [33]. They used a mixture of decane and undecane as the "oil" to match the viscosity of water, eliminating the need to figure out how much of the bead was in the water, and how much was in the oil. The "effective viscosity" of the interface remains a question.

Sickert and Rondelez found the shear viscosity of Langmuir monolayers of surfactants on an air-water interface by measuring the diffusion of a very dilute solution of polystyrene microspheres [38].

In 2008 Bum Jun Park published a study on the interaction forces between colloidal particles on an oil-water interface [9]. The effects of different concentrations of salt, i.e. ions, and surfactant, on the forces, were found using multiple optical traps to hold beads still relative to each other. Time-dependence of the interactions was observed. 


\subsection{OPTICAL TRAPPING THEORY AND SET UP}

In this chapter, the basic optical forces will be explained. The experimental set-up will be given, making it clear what goes into determining the trap dynamics. In order to quantify the interaction force between the beads, the trap must be actuated, the displacement of the beads from the trap must be sensed, and these instruments must be calibrated.

\subsection{OPTICAL TWEEZER THEORY}

Refraction of light transfers momentum from photons to the objects with which they interact. This force is large enough to counteract forces due to gravity, viscous drag, etc. on a microparticle. It is important for the beam to have a high intensity gradient if three dimensional trapping is desired. This can be obtained by filling the input aperture of the trap with the laser enough that the convergence angle is high while using a high NA lens [5]. The convergence angle is the angle the light makes with the optical axis as it reaches the focus (see Figure 3).

When a photon encounters an object, it can be either refracted or absorbed. Conservation of momentum demands that the object interacting with the light has its own momentum changed if the momentum of the photon is changed. The momentum, $p$, of a photon is:

$$
p=\frac{h[\mathrm{~J} \cdot \mathrm{s}]}{\lambda[\mathrm{m}]},
$$

where $\lambda$ is the wavelength of light and $h$ is Planck's constant. The force due to the photons is their rate of energy transfer (power) divided by their velocity:

$$
F=\frac{P n \hat{k}}{c}
$$


where $P$ is the incident power, $n$ is the refractive index of the propagating medium, and $\hat{k}$ is the vector pointing in the direction of the beam [44]. It is shown below that a laser beam can provide enough photons to move small objects if some of this momentum is transferred to the object through interaction. If the light goes through the particle without refracting, reflecting, or absorbing, there is no change in momentum, and no force generated.

For example, consider a red HeNe laser $(\lambda=633 \mathrm{~nm})$. It has a power, $P=5 \mathrm{~mW}$. The maximum force it could exert on a bead if all of the momentum were transferred from the photons to the bead:

$$
\begin{gathered}
F=\frac{\left(5 \cdot 10^{-3} \mathrm{~J} / \mathrm{s}\right)(1.33)(1)}{3 \cdot 10^{8} \mathrm{~m} / \mathrm{s}} \\
F=1.6667 \cdot 10^{-11} \mathrm{~J} / \mathrm{m}=16.667 \mathrm{pN}
\end{gathered}
$$

The $n$ chosen for the calculation is $n_{\text {water }}=1.33$. A focused laser beam causes two forces on a dielectric sphere, a scattering force, and a gradient force. Of the momentum that is transferred, some goes into creating the scattering force and some goes into creating the gradient force, so the force on the bead in any one direction is less than this maximum value. The weight of the bead is on the order of fempto-newtons, three orders of magnitude lower than this maximum possible force generation. The amount of force per unit of input power is known as the trapping efficiency, $q$.

$$
q=\frac{F_{\text {trap }}}{\frac{n P_{\text {laser }}}{c}}
$$

where $\mathrm{n}$ is the relative refractive index.

In the research, the laser beam will travel through 5 different media being refracted and reflected along the way. The beam starts in air $(n=1.00)$, then travels through the glass of the coverslip $(n=1.52)$, into the layer of water $(n=1.33)$, into the polystyrene bead $(n=1.59)$, to the mineral oil $(n=1.47)$, and finally back out into air. The values given are the real parts of the refractive index of the five materials. The imaginary parts of the refractive indices are zero for all five.

If the particles have a higher index of refraction than the surrounding medium, they are attracted to the beam, while particles with lower respective refractive indices are pushed out 


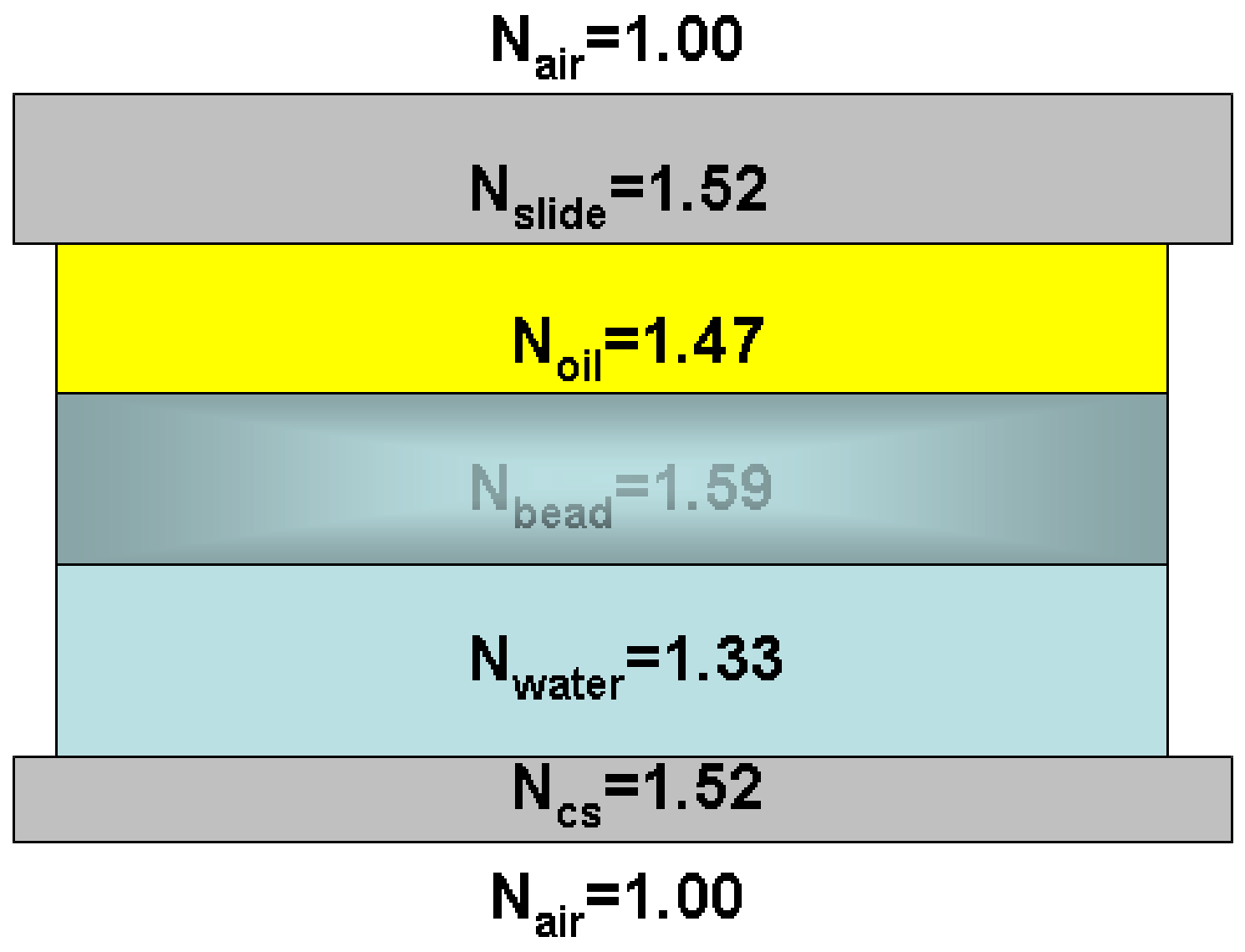

Figure 4: The different refractive indices that the beam encounters as it makes its way through the sample. 
from the beam. The refractive index of both water and oil are less than polystyrene, so the qualitative effect will be the same as if the microspheres were in bulk water or bulk oil. This is important to the experiment because different particles react to the beam differently.

\subsubsection{Mie Scattering}

The fundamental way in which the photons interact with matter depends on the characteristic size of the material. There are two limiting cases, $\lambda \ll d$ and $\lambda \gg d$, where $d$ is the dimension of the object along which the light is interacting.

Mie scattering includes all light scattering, of which Rayleigh scattering is a special case [23]. Geometrical (ray) optics is based on the assumption that all the pertinent dimensions of the materials that the light is interacting with are large compared to the wavelength $(\lambda \ll d)[26]$.

The force exerted on a bead is dependent not only on the power of the beam, but also the relationship between the laser wavelength and the size of the bead. When the wavelength matches a surface wave resonance, it takes more laser power to apply the same trapping force [6].

Because the particles in this experiment are 2 microns in diameter, neither the ray optics nor the Rayleigh regime explicitly applies, but it has been shown that single beam gradient force traps are stable in the transition regime between Rayleigh and Mie scattering [1]. The particles used in this experiment are in the transition region between the Mie and Rayleigh regimes. The light scattering can be described by the generalized Lorenz-Mie theory (GLMT) [46].

In Figure 5, there is an explanation of the three cases of relative bead/beam focus position in the ray-optics regime. The vector force exerted on the bead is equal to the difference between the incident momentum vector, and the sum of momentum vectors from all the scattered light coming out of the bead. Most of the momentum transfer comes from the change in direction of the emergent rays [1]. This explains why the resultant force matches the opposite direction of the refraction of the emergent rays. 


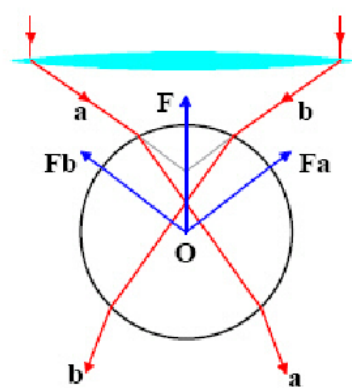

(a)

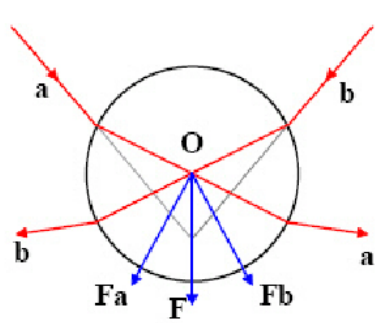

(b)



(c)

Figure 5: If objective lens has a high enough NA, the bead always feels a force restoring it to the focus of the beam. a.) Shows that a bead beyond the focus of the beam, is pulled backward. b.) Shows that a bead centered behind the focus is pushed forward. c.) Shows that a bead that is to the right of the focus, is pushed to the left [45]. 
It is valid to use ray tracing-geometric optics when the value of a dimensionless size parameter, $\beta$ is greater than 100: [44]

$$
\beta=\frac{2 \pi n_{2} R}{\lambda}
$$

where $n_{2}$ is the index of refraction of the liquid, $\mathrm{R}$ is the radius of the microsphere and lambda is the wavelength of laser light in a vacuum. In the case of this experiment, $\beta$ is about 8 . The ray optics method of determining the forces is within a factor of 2 around this range of $\beta$ values [44].

$$
\frac{2 \pi(1.33) \cdot 10^{-6}}{1.064 \cdot 10^{-6}}=7.85
$$

\subsubsection{Optical Trap set-up}

The optical trap set up can be seen in Figure 6. A Zeiss Axiovert 200 inverted microscope acts to image the particles and collect light to be analyzed. The fast-steering mirror (FSM) used to control the location of the beams is an FSM-300 from Newport. The laser used to create the trap is a Compass 1064-2500 MN diode-pumped Continuous Wave IR laser from Coherent. The wavelength is $1064 \mathrm{~nm}$. This wavelength was chosen because it does not do as much damage to biological samples which will be used in future experiments.

The beam from the laser comes out with a divergence that can be accounted for to provide a collimated beam. To counteract the divergence and expand the beam, a telescope is placed a few centimeters beyond the laser. A half-wave plate is used to adjust the polarization of the beam. The beam must have a 45 degree orientation so that each beam is of equal intensity after the polarizing beamsplitter. Actually, because the mirror on the FSM has a lower reflectivity in IR, the half-wave plate can actually adjust the polarization so that both beams have the same intensity at the image plane. This can be determined with a power meter.

The half wave plate at the beginning of the optical train determines how much power goes to the stationary mirror and the FSM. The wave plate shifts the polarization of the laser light by adding different phase delays to different incoming polarizations. It has two axes, a slow and a fast axis. By placing the wave plate in a rotational mount, it is possible to change the 
angle at which the two axes are oriented with respect to the incoming light. This allows for the outgoing light to have a continuum of polarizations from completely vertically polarized to completely horizontally polarized and anywhere in between. The polarizing beam-splitter further down the optical train splits the light according to polarization, deflecting vertically polarized light and allowing horizontally polarized light to pass through. The response of the QPD is heavily dependent on the half-wave plate position. If there is more power going to the vertically polarized beam (FSM) then there is a large response, but if most of the power is in the stationary beam, very little signal is found from scanning the FSM beam across a microsphere.

The beam is then raised to the level of the entrance to the microscope with a periscope. After the periscope, the beam is divided by a polarizing beamsplitting cube. One beam is directed to the FSM while the other beam is directed to the stationary gimbal-mounted mirror. The FSM steers the beam that will control the moveable bead. The gimbal mounted mirror will hold a reference bead steady. Now both beams are recombined by another polarizing beamsplitter. This "double" beam is sent through another lens.

There are reasons for the specific spacings between each lens and mirror in the system. For example, it is important for the FSM and stationary mirror to be imaged to the entrance aperture of the microscope objective, so that the tilt of the mirror only changes the angle of the light incident on the microscope objective, not the position. This means that the beam will only translate, and not change in angle at the specimen plane. 


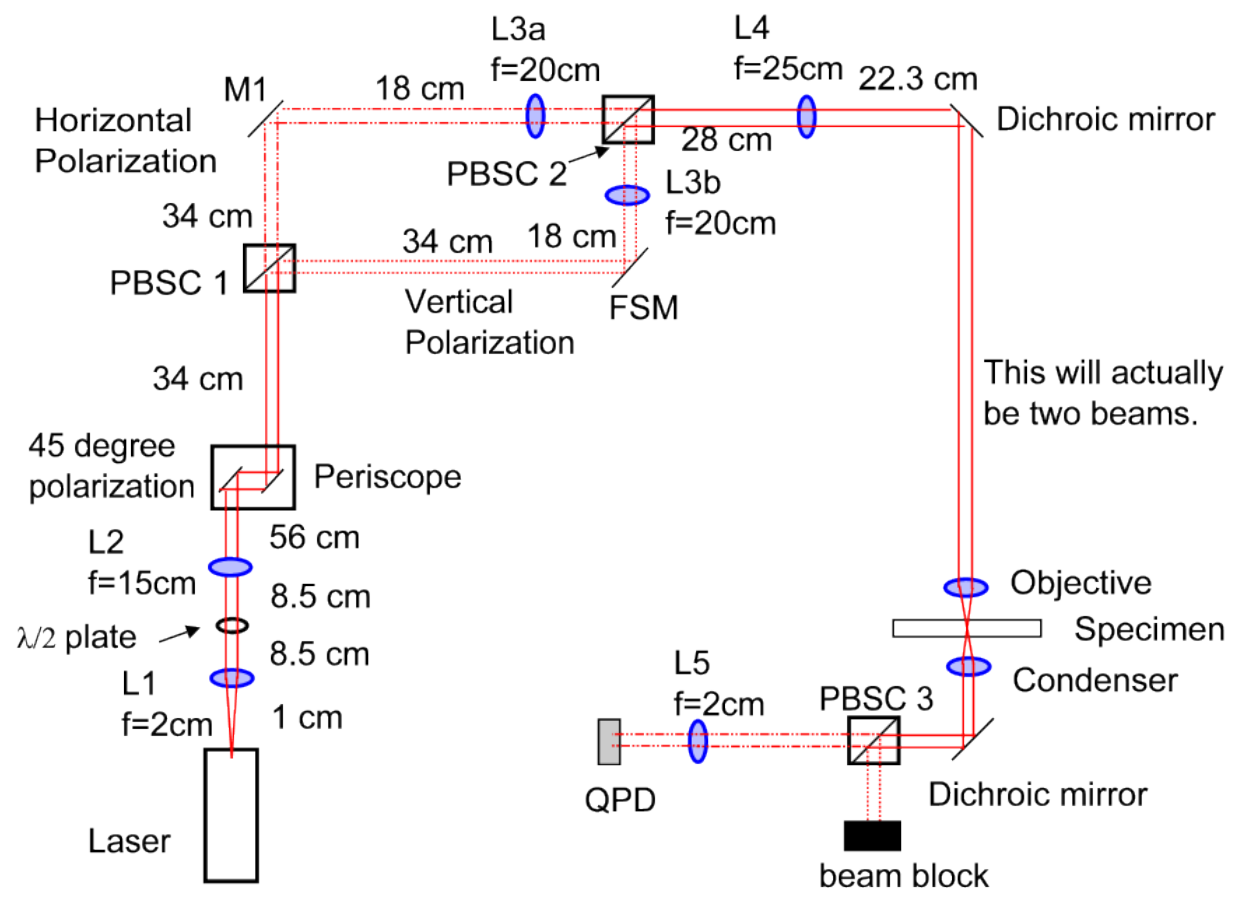

Figure 6: Optical Tweezer Setup with Gaussian beam. 
Viscosity has been found to decrease with increasing laser power as the sample heats up because the particles absorb some energy and transfer it to the surrounding liquid [2]. In Bishop et al. they report a decrease in viscosity after $0.1 \mathrm{~W}$ at the sample, but this is significantly higher than the power at the sample in this experiment $(\sim 2 \mathrm{~mW})$. The Gaussian trap experimental set up takes up about 12 square feet on an optical table. This could probably be optimized to about 10 square feet. The part where the beam comes up and gets reflected to the QPD is shown in Figure 9. Figure 7 shows the variables that are measured in the experiment. According to Crocker and Grier, the trapped particles do not appreciably heat up (less than $0.1 \mathrm{~K}$ for $30 \mathrm{~nm}$ diameter spheres) because the light is not strongly absorbed and water carries heat away quickly [11]. This does not match with the fact that the interface flow increases significantly when the laser is turned on.

The equation of motion for the bead in the trap:

$$
m \ddot{u}+c \dot{u}+k u=0
$$

where $\mathrm{m}$ is the mass of the bead, $\mathrm{c}$ is the damping due to the viscosity of the medium, and $\mathrm{k}$ is the stiffness of the trap $(\mathrm{u}=\mathrm{x}-\mathrm{z})$. The variables in the system include $\mathrm{u}$, the distance the bead is from the center of the trap, $\mathrm{z}$, the distance the trap is from a reference point, and $\mathrm{x}$, the absolute position of the bead with reference to the microscope. The term $\mathrm{u}$ is necessary because the spring force is with respect to how far the bead is from the center of the trap, but the damping from the water occurs no matter where the bead is.

In this case, the damper is the Stokes drag of oil and water and other forces caused by the interface between oil and water. The equation for Stokes drag of a sphere in bulk:

$$
F=6 \pi v \mu R
$$

where $v$ is the velocity of the particle with respect to the fluid, $\mu$ is the viscosity of the fluid and $R$ is the radius of the particle. This equation is not explicitly valid for a bead on the interface because the effective viscosity of the interface is not known. Also, the bead could potentially be rolling, again changing the concept of "viscosity". Depending on where the bead sits on this interface (i.e. how much surface area is in the water or the oil) changes the damping properties of the system. 


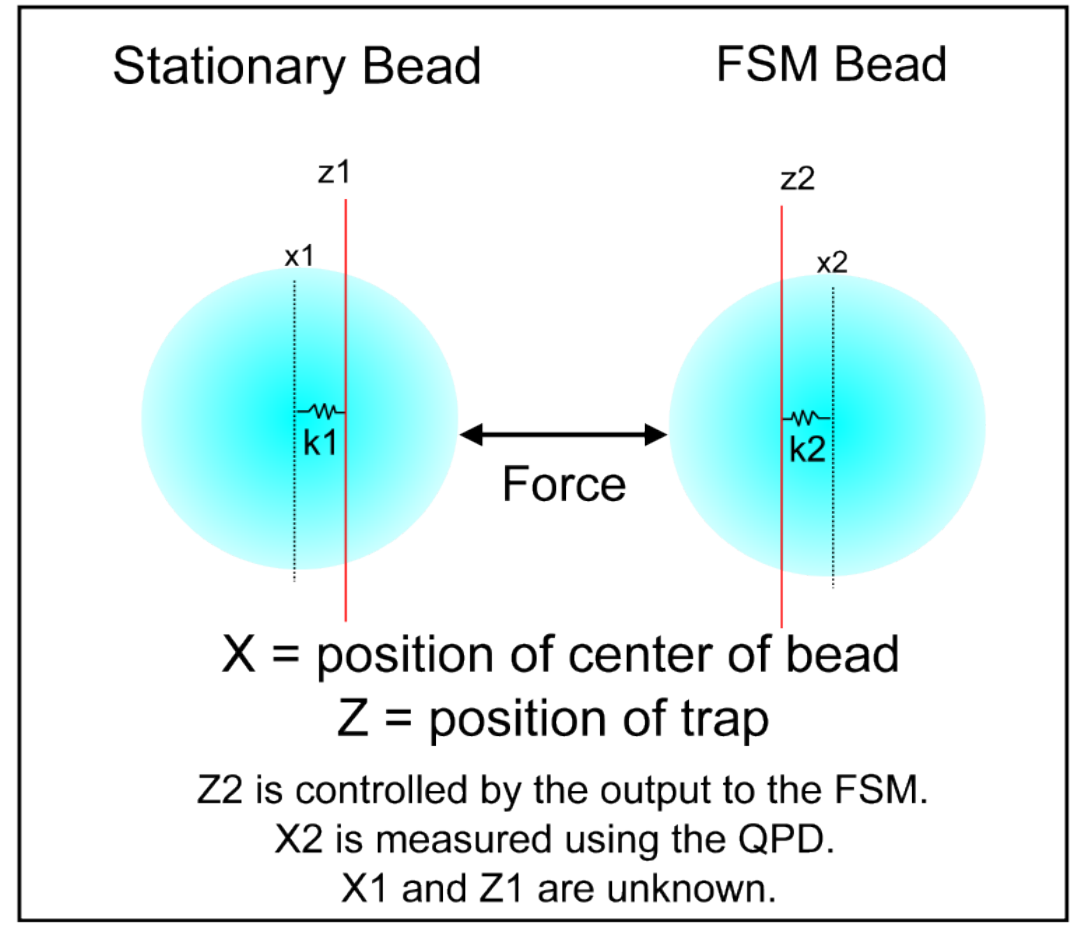

Figure 7: Schematic of the variables used to calculate the force between the beads. 


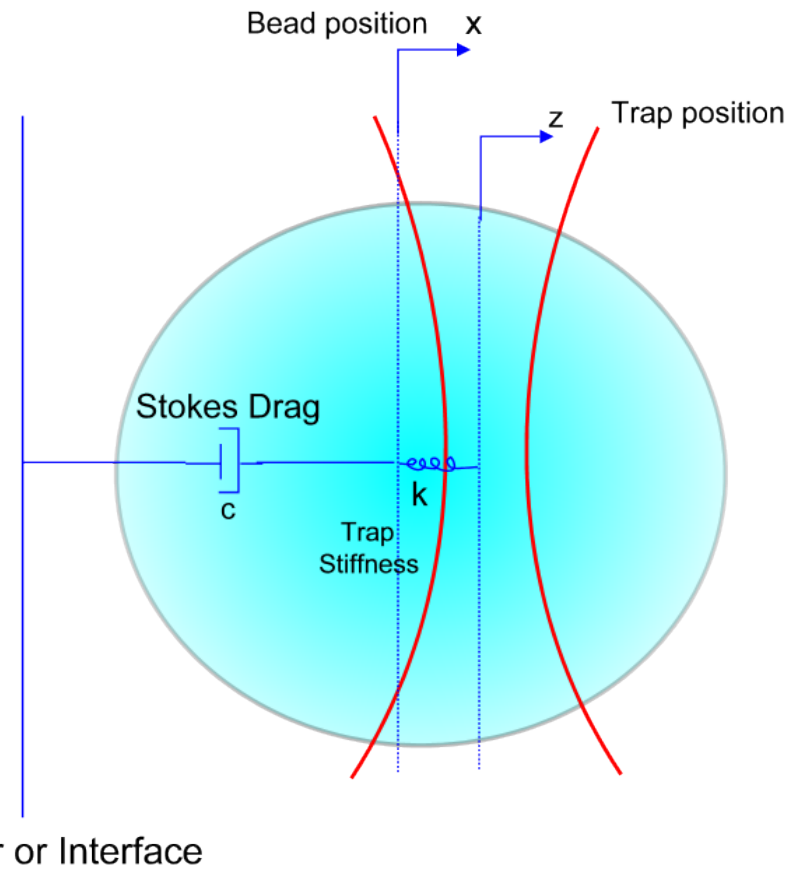

Figure 8: Free body diagram of forces on bead. The equation of motion can be derived from this. 
A dichroic mirror reflects the IR light through an LD Plan Neofluar 63x objective (NA $0.75)$ to the specimen. The objective has a corrective collar that allows correction for coverslip thickness. The only reason a higher NA would be useful is for the collection of light onto the QPD. The light proceeds to be collected by a condenser (NA 0.55). Another dichroic mirror reflects the laser light to another polarizing beam splitting cube. The beam corresponding to the stationary bead is reflected and blocked. The beam corresponding to the moveable bead is sent through a collimating lens. This beam is then analyzed using a Dual Axis position sensing diode (PSD) Sum and Difference Amplifier from Pacific Silicon Sensor Incorporated. The device is called a quadrant photodiode (QPD) from this point on. A small active area was chosen to minimize the amount of dark noise coming from the parts of the diode which the scattered beam is not filling [36]. The small active area also allows for less magnification, meaning that the QPD can be placed closer to the microscope, and any vibrations of the platform on which it stands, have less of an effect on the signal. The differential voltage of the left and right sides $(\mathrm{X})$ and top and bottom halves $(\mathrm{Y})$ are measured along with the total voltage detected by the $\mathrm{Y}$ diodes. The total voltage can be used to normalize the readings because the laser intensity has been found to pulse at about $170 \mathrm{~Hz}$.

The images that are analyzed for experimental data have fundamental limits in resolution. This has to do with the CCD (Hitachi KP-D20 A/B) specifications shown in Table 1.

Table 1: Specifications of CCD camera.

\begin{tabular}{ll}
\hline Total pixels (NTSC) & $811(\mathrm{H}) \times 508(\mathrm{~V})$ \\
\hline Effective pixels & $768 \times 494$ \\
\hline Scanning Area & $6.45 \mathrm{~mm} \times 4.84 \mathrm{~mm}$ \\
\hline Unit Cell Size & 8.4 microns x 9.8 microns \\
\hline
\end{tabular}

Most times that measurements are made with optical traps, the object under test is within microns of the objective, allowing for the use of a high NA objective. In the case of this experiment, the beads are over one millimeter from the objective. This leaves two 




Figure 9: Sensing Portion of Set-up. 
choices, lower magnification or lower NA. The magnification is needed to easily distinguish between particles on the video screen. Lower NA is allowable because three dimensional trapping is unnecessary.

The reduced NA of this lens reduces the stiffness of the trap. There is a corrective collar to adjust to different coverslip thicknesses. This experiment uses a coverslip that is $0.17 \mathrm{~mm}$ thick. The actual depth into the water does not need to be accounted for with the collar.

\subsection{SYSTEM CHARACTERIZATION}

It is important to know that the sensing and actuating equipment are fast enough and sensitive enough to detect the movement of a bead within the beam. The peak and roll-off in the dynamics of the FSM are around $1 \mathrm{kHz}$. The roll-off of the QPD dynamics are around $10 \mathrm{kHz}$. Both of these are far above the $100 \mathrm{~Hz}$ maximum signal that will be measured in the calibration and pair potential experiments. Brownian motion will be randomly putting forces on the bead in every direction. The laser, microscope, etc. will all be vibrating due to ambient noise, people walking in the halls and air currents in the room. The FSM has noise associated with it. These noise sources are not significant to the finding of force vs. position values.

The trap will consist of a stationary beam with horizontal polarization and a steerable beam with vertical polarization. The stationary beam will hold one particle still while the other particle is moved with respect to it. Both beams are assumed to be of equal efficiency meaning that the stiffness is only a function of input power.

The Reynolds number that most microspheres experience is very low because of the small length scale. This means that viscous forces dominate over inertial forces. When the beam is dragging the bead somewhere, it will not overshoot the stop position when the beam stops moving.

The QPD measures how far out of the trap center the bead is at a certain time, and this information is fed through the dSpace board. The dSpace board is connected to a Simulink model of the system that is run through Matlab. 
In order to characterize the optical tweezer, a random noise source is used as the input to the FSM. The voltage signal controlling the FSM is put through an anti-aliasing (AA) filter because there is a finite sampling rate.

The laser is placed through a bead that is stuck to the coverslip so that only the noise source is acting upon the QPD. The response of the QPD is then recorded and analyzed using matlab. A bode plot of the power spectral density allows the FSM dynamics to be characterized. The FSM dynamic model is used to make a prediction of where the bead is with reference to the microscope.

dSpace provides the control signal to the FSM. Because the control is in discrete time, the mirror moves in jerky steps. The physical mirror "rings" in response to these steps, adding extra noise to the measurement. One way to smooth the steps that the mirror makes is to place a smoothing filter between the dSpace control signal, and the FSM mirror signal input. The smoothing filter is a simple RC circuit with the cutoff frequency designed to be a little below the Nyquist frequency of the control signal $(400 \mathrm{~Hz})$.

\subsection{ACTUATION BY FAST-STEERING MIRROR}

The fast-steering mirror is a voltage controlled device. A certain voltage input to the faststeering mirror (FSM) will cause the mirror to tilt on the $\mathrm{x}$ and/or $\mathrm{y}$ axis. By placing the FSM at the front focal plane of the beam, it is possible to translate the beam across the specimen plane without changing the tilt of the beam. This means the beam always leaves the objective going straight, perpendicular to the specimen plane.

\subsection{SENSING}

In order to measure force exerted on the bead by the laser beam, several sensors must be used.

There are three things that can be physically measured in the experiment, the "distances between" and "sizes of" objects in the specimen plane, and two sets of voltages, the signals 
controlling the fast-steering mirror's two axes, and the voltages coming form the quadrant photodiode. Two quantities are needed to determine the force experienced by a bead on the interface, the displacement, $x$, and the trap stiffness, $k$.

All the images of the beads used in this thesis are taken using a CCD camera attached to the microscope. The images are helpful in calibration. The laser beam is guided along the optical path by lenses and mirrors. Almost all of these are stationary. The trap can be moved by moving a lens and/or a mirror. In order to find the force between two particles with respect to their distance from each other in a systematic way, it is useful to have a mirror that is computer controlled. Also, the light that is scattered by the displacement of the bead from the center of the trap must be measured by a light sensitive device, in this case a QPD. The transfer functions between all the sensors and actuators must be determined through calibration steps. Then the data can be interpreted.

\subsubsection{Quadrant Photo Diode (QPD)}

A photodiode converts incident photons into current. The concept of a quadrant photodiode is that four diodes can have their photocurrents measured simultaneously and compared. For example, if there is a beam of light centered on the four panes of the photodiode, the difference in current between the sum of the right two panes and the left two should be zero. Also, the difference between the sum of the top two and the sum of the bottom two panes should be zero. The XSUM and YSUM signals are the sums of the two sets of photocurrents. The "sum" signals can be used for normalization. There is an area in the middle of the active area where the response to position is linear. All frequency dependent measurements should be taken in this linear region. A visualization of how the QPD creates signals is shown in 10 The experiment involves the use of a self-aligned sensor. The QPD is placed at the Fourier plane, meaning the spot on the QPD face never moves. For example, the conjugate action of whatever happens at the specimen plane happens at the QPD. If the beam is moved across the specimen plane with the FSM, there should be no change at the QPD because this will only change the angle of light hitting it. If the beam is stationary, and the trapped bead moves relative to the beam, the light will be scattered to a different angle. This will cause 


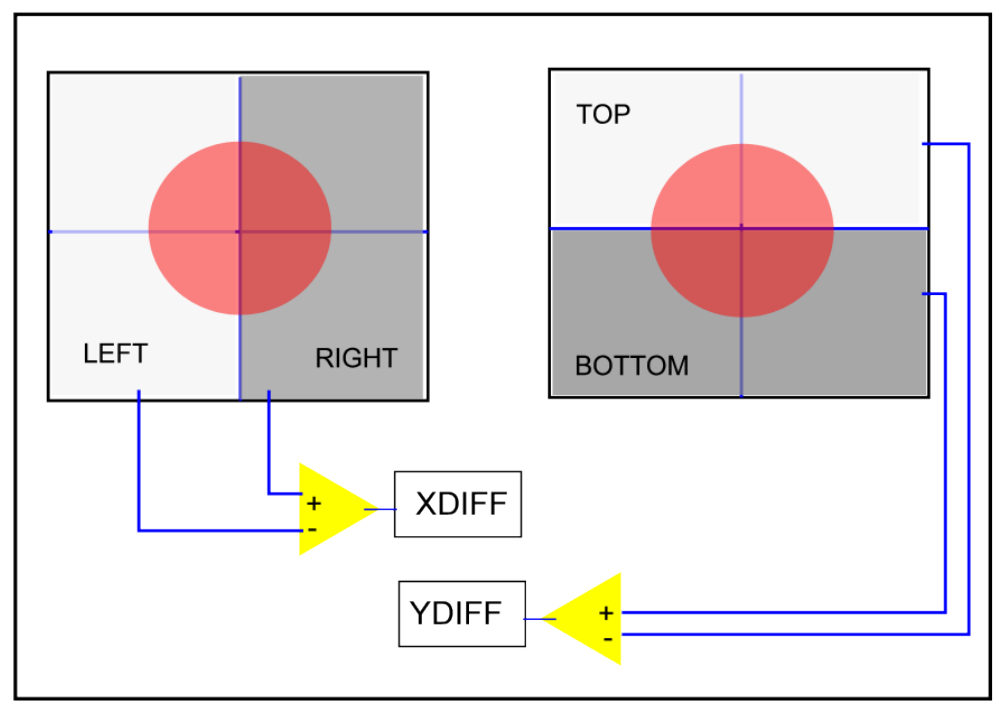

Figure 10: Two of the most important signals coming from the qpd are the XDIFF and YDIFF signals. The XDIFF signal is the difference in current between the right and left half of the qpd. The YDIFF signal is the difference in current between the top and bottom half of the qpd. 
a change in the intensity distribution at the QPD (see Figure 11). If a bead is trapped and scattering light, the intensity of different parts of the circle of light on the QPD changes, but the whole circle never translates across the face of the QPD. Figure 12 shows the how misalignment of the QPD system can lead to a bias in the voltage reading as the bead moves relative to the laser. To prove that the $\mathrm{QPD}$ is at a plane conjugate with the objective, a coherence measurement can be made.

\subsection{CALIBRATION}

In order for the voltages measured by the QPD to have any real meaning, the system of FSM, trap, bead, and QPD must be calibrated and the transfer functions between them, found. There are two main variables in the experiment, the displacement of the bead from the center of the trap, and the stiffness of the trap. The first two subsections of this section will cover the former. The last subsections will cover the stiffness determination.

\subsubsection{FSM volts to Trap displacement}

The voltage signals sent to the FSM cause the trap to move across the specimen plane. In order to calibrate the voltage to the trap displacement, an image of the trap is taken at two extreme voltages, i.e. $(-3 \mathrm{~V}$ and $+3 \mathrm{~V})$. The six volt span is correlated to a distance by measuring how many pixels wide a known 2 micrometer diameter bead is. The bead is measured 10 times and an average number of pixels is found. Then the distance the trap traveled in the 6 volt swing is measured in pixels, and converted to micrometers. The results of the calibration are given in Table 2 . 


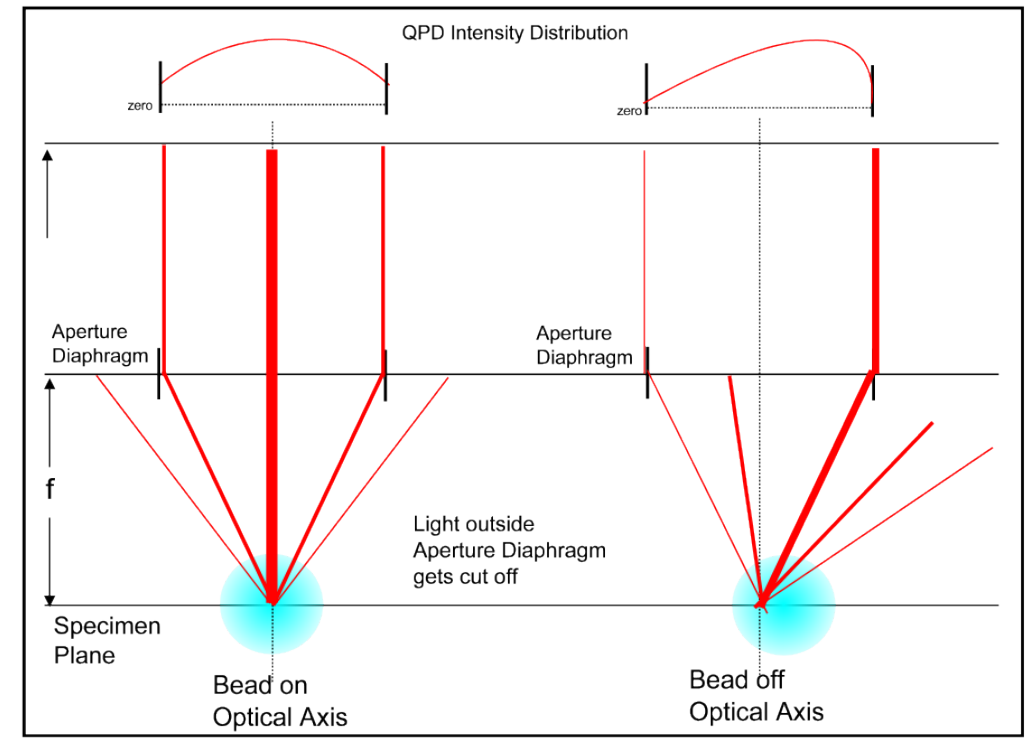

Figure 11: Schematic representation of how a change in relative bead/beam position does not move the spot on the QPD, but does change the position of the intensity maximum, allowing for displacement measurement. The spot on the QPD does not move because the specimen and QPD are at conjugate Fourier planes and the aperture diaphragm cuts off light that would be projected to a different point on the QPD. 


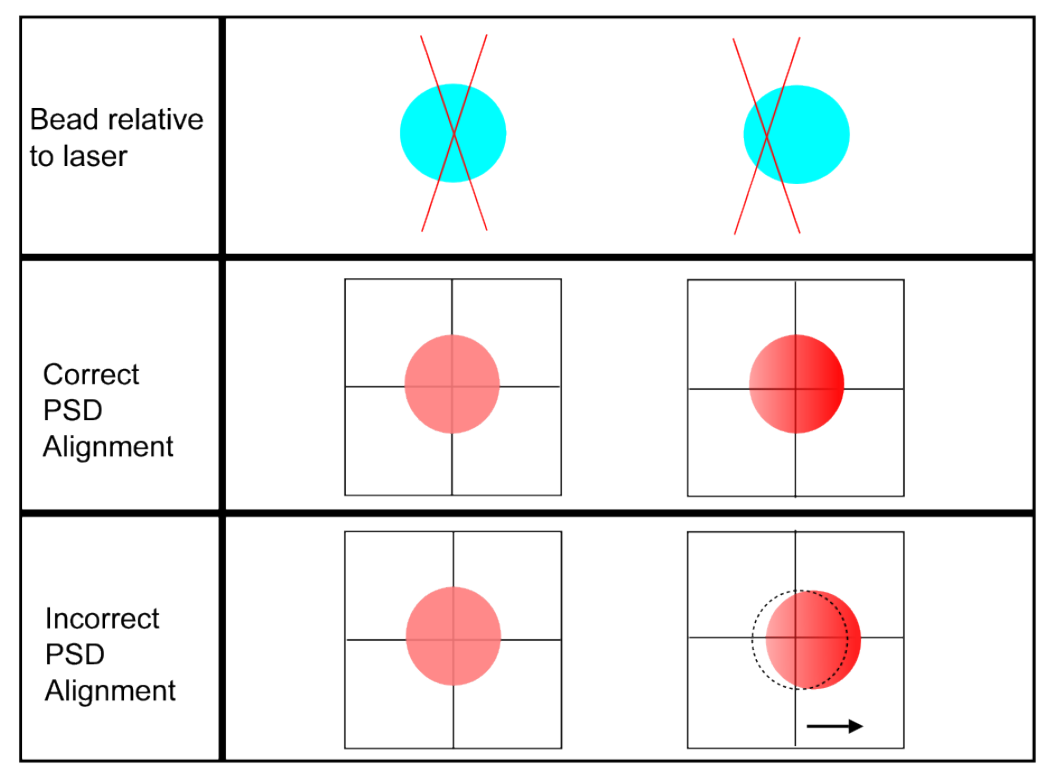

Figure 12: The middle row shows the same thing as the previous figure, but the third row shows the effect of putting the QPD somewhere besides the back focal plane (BFP) of the condenser. 
Table 2: Conversions in Force Calculation.

\begin{tabular}{l}
\hline $1.6 \mathrm{~V} / \mu \mathrm{m}$ for $\mathrm{Y}$ \\
\hline $2.1 \mathrm{~V} / \mu \mathrm{m}$ for $\mathrm{X}$ \\
\hline
\end{tabular}

It is expected that the FSM Volts per pixel (see Figure 13) moved across the microscope screen should be the same for all cases from beads dried on slides, to beads trapped in bulk, to beads trapped at an interface. Measurements have determined a maximum deviation for the Gaussian trap case. Table 3 shows the relationship between FSM volts and pixels the beam moves across the screen in the horizontal and vertical directions for just a slide with water and an actual interface. The thickness of water that the beam travels through in the "bulk water" case is approximately 100 microns while in the "interface" case, the beam travels through about 500 microns of water. The fact that both cases are within 5 percent of each other implies that the beam is going straight through the sample at all FSM voltages tested. There are only small angles introduced to the beam.

Table 3: FSM volts per pixel of beam movement across the screen for the Gaussian trap in bulk water and on the interface.

\begin{tabular}{ll} 
Case & FSM volts per pixel \\
\hline Bulk water Horz. & $0.032 \pm 2 \times 10^{-4}$ \\
\hline Interface Horz. & $0.032 \pm 3 \times 10^{-4}$ \\
\hline Bulk Water Vert. & $0.022 \pm 2 \times 10^{-4}$ \\
\hline Interface Vert. & $0.021 \pm 2 \times 10^{-4}$
\end{tabular}

\subsubsection{Displacement of the bead from the trap}

The FSM volts to micrometer conversions can then be used in interpreting data from scanning the beam across a stationary bead. The reaction of the quadrant photodiode (QPD) to the scattered light can be correlated with the exact position of the beam with respect to the 


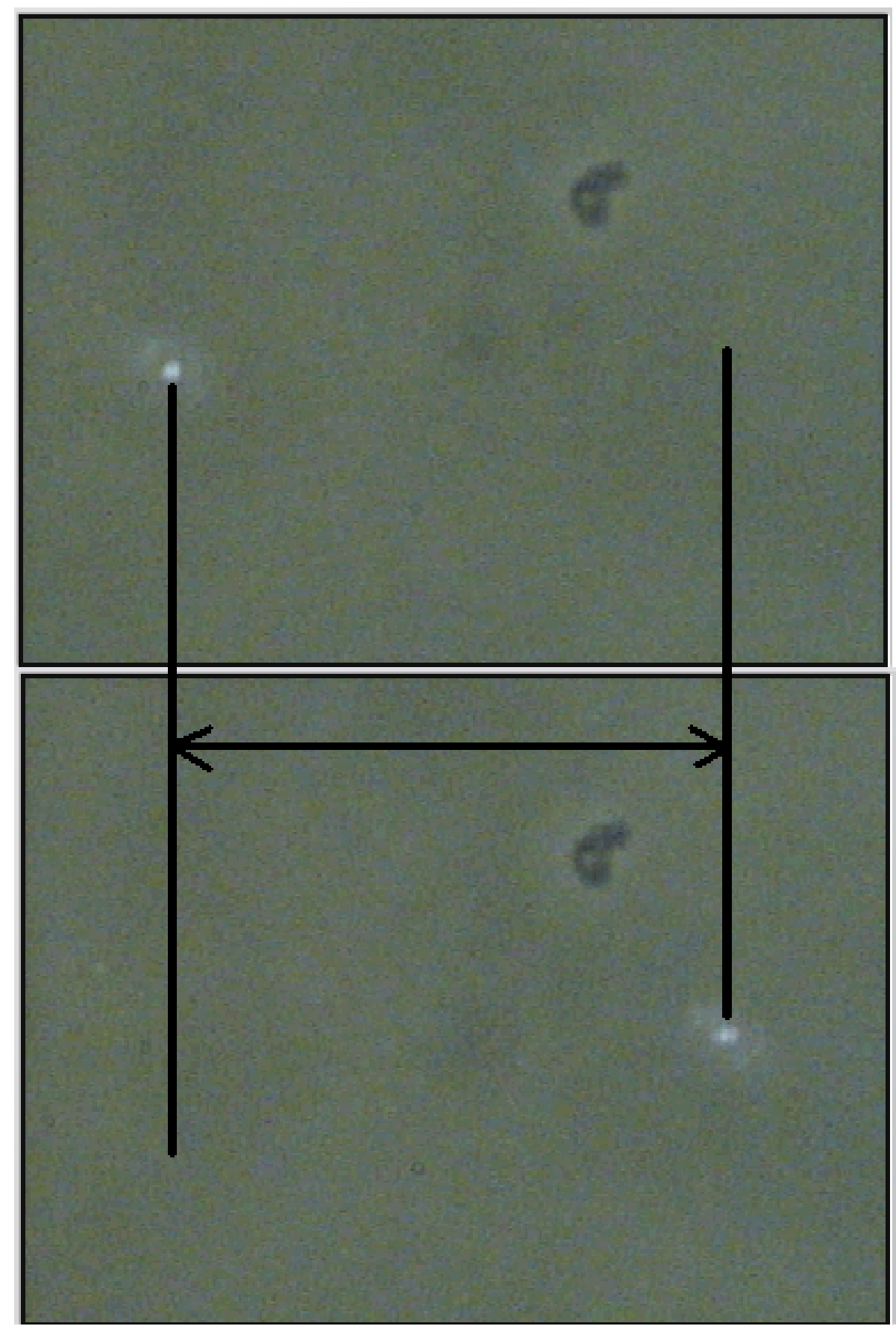

Figure 13: Here is an example of an FSM volts to nanometer calibration. The number of pixels in the image between the two extremes (a 6 volt span) in FSM voltage is found. This is compared to the number of nanometers per pixel, found by measuring the known diameter of the image of the microsphere. 
bead at all positions. A certain displacement of the beam from the center of the bead, or vice versa, will cause a certain voltage response by the QPD. By drying beads onto a slide and scanning the laser beam across a stationary bead, this transfer function can be found.

In order to find the transfer functions between the voltages measured and their corresponding forces and displacements, several control experiments must be done. The experiments were discussed in general in Chapter 2, but now the details will be given.

In this thesis experiment, the laser must go through many different media. This can complicate the meaning of the light scattering that that happens between the microscope objective and the QPD. To help resolve what is going on, it is helps to find the effect of light scattering off a bead in only water, and a bead in only mineral oil. Drying the beads to a coverslip ensures that the trap does not move the bead, then the beam can be rastered across the bead in a controlled manner. Whether the laser light goes through the liquid, and then hits the bead, or goes from the bead into the liquid, will also be investigated, covering both cases of what light will be doing at the interface, and their opposites for comparison. These measurements will provide a contour plot that corresponds to the QPD voltage at all points of the bead. This type of measurement (raster of stationary bead) will enable the determination of how far a bead is pushed out of the center of the beam by an external force. Specifically using dried-on beads is only necessary as a first-time sanity check to make sure the QPD is functioning properly.

Figures 14 and 15 show the QPD voltage corresponding to the beam sweeping across the bead. The bead has been dried on the coverslip, and covered with water. The center of the bead is about zero, and the edges cause the largest signal. The slope between the maximum and minimum value is important. The figures are qualitatively the same for all cases, water, oil, or interface.

In order to interpret the interaction force measurements at the interface, it is necessary to take a bead contour of a bead while it is on the interface. It is impossible to "dry" a bead to the interface, therefore the raster must be done with a very weak beam while the bead is being trapped by a significantly stronger beam.

The dried bead contour calibration measurements were performed at two power levels to confirm that there is a proportionality between the QPD response at different laser powers. 


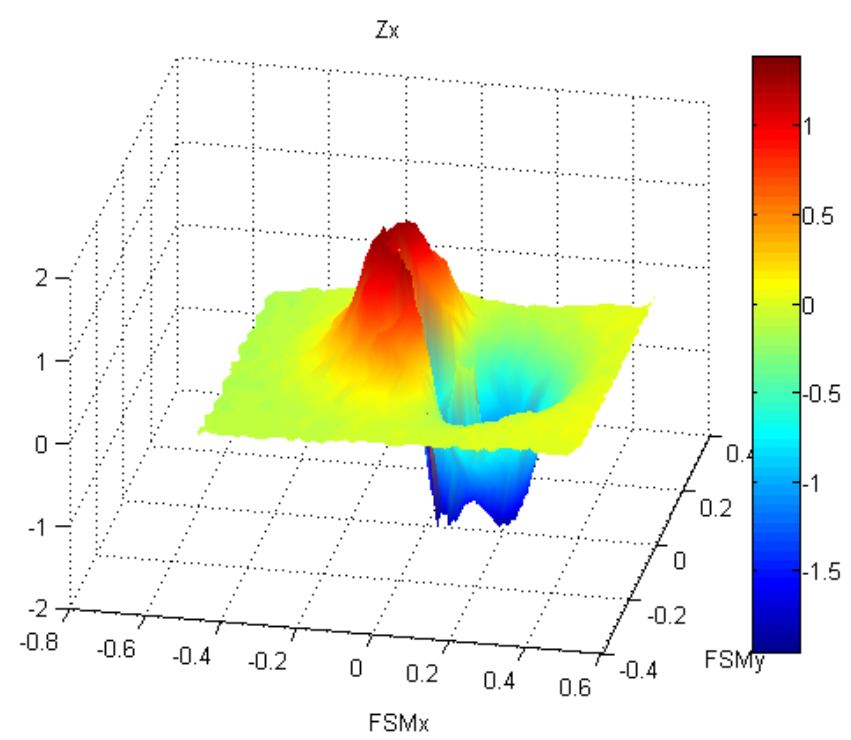

Figure 14: This is the QPD voltage from X channel of a bead dried to a coverslip and covered in water. The trap was rastered across the face of the bead.

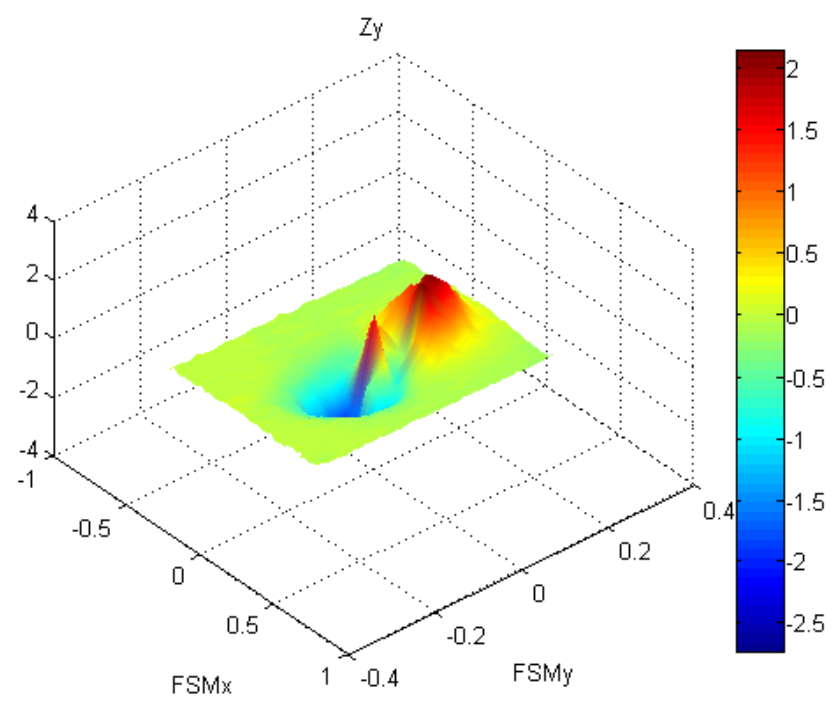

Figure 15: This is the QPD voltage from Y channel of a bead dried to a coverslip and covered in water. The trap was rastered across the face of the bead. 
Table 4: The table shows the average dynamic range of QPD response to scanning the laser across a fixed bead. This shows that the ratio between trap power and low power is much more important than the combination of media.

\begin{tabular}{lll} 
Slide Oil Low & 0.1625 & \\
\hline Slide Oil Trap & 0.91 & 5.6 \\
\hline Slide Water Low & 0.26 & \\
\hline Slide Water Trap & 1.48 & 5.7 \\
\hline Coverslip Oil Low & 0.17 & \\
\hline Coverslip Oil Trap & 1.02 & 6 \\
\hline Coverslip Water Low & 0.21 & \\
\hline Coverslip Water Trap & 1.25 & 5.95
\end{tabular}

One level will be designated "LOW" and is used to raster scan the beads when they are trapped on the interface by a higher power stationary beam. The other power level will be designated "TRAP" and will be the power of the beam during the actual interaction force measurement.

The "LOW" power was $30 \mathrm{~mW}$ while the "TRAP" power was $180 \mathrm{~mW}$. If the dynamic range of the QPD measurement, i.e. the difference between the maximum and minimum voltage on any particular scan is proportional to the laser power, it would differ by a factor of six. The fact that it is means that data taken at "LOW" power in bulk raster scans can be extrapolated to correlate with data taken at "TRAP" power. 


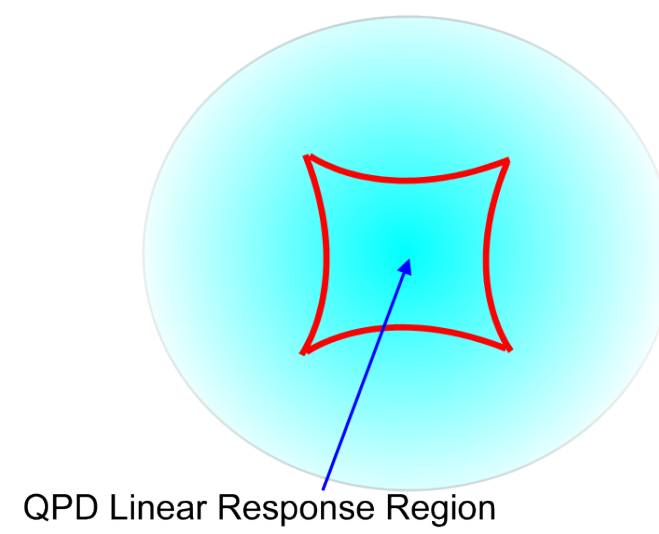

Figure 16: The QPD voltage response is only linear in the central region of the bead. All measurements must take place with this small displacement from the bead center.

Figure 17 shows a slab approximation of the difference in light scattering between oil and water, to explain the difference in QPD response for beads dried on the coverslip. Figure 18 shows the difference in light scattering between oil and water, to explain the difference in QPD response for beads dried on the slide. A Matlab model of the scattering for the four cases gives a maximum difference of 2 percent. This does not match with the measured data shown in Table 4. Possible explanations are that the oil absorbs more light than water, or that the spherical nature of the particles has a large effect on the scattering, and the slab approximation is not appropriate.

It has been found that the slope of the linear region of the response curve is very different depending on whether the dried bead is covered with water or oil. This probably has to do with the difference in relative refractive index. The difference in refractive index, between water and polystyrene is larger than the difference between mineral oil and polystyrene. The slope of beam position to QPD volts is much greater for the case of water than oil. 
HIGHER dynamic range



HIGH CONTRAST

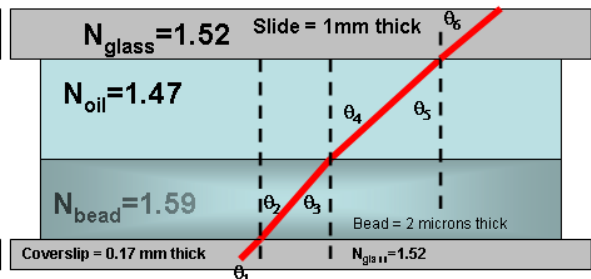

Figure 17: Dry beads onto two cover slips. Make one slide with water and one with oil to test the response of the QPD as the beam is scanned across the bead and diffracted. Treating the bead like a slab of polystyrene, ray goes through two refractions. Say that $\theta_{1}=15$ degrees. Both cases give the same resulting angles, but because $\theta_{4}$ is greater in the water case, the displacement of the beam is further from the entrance point than in the oil case.
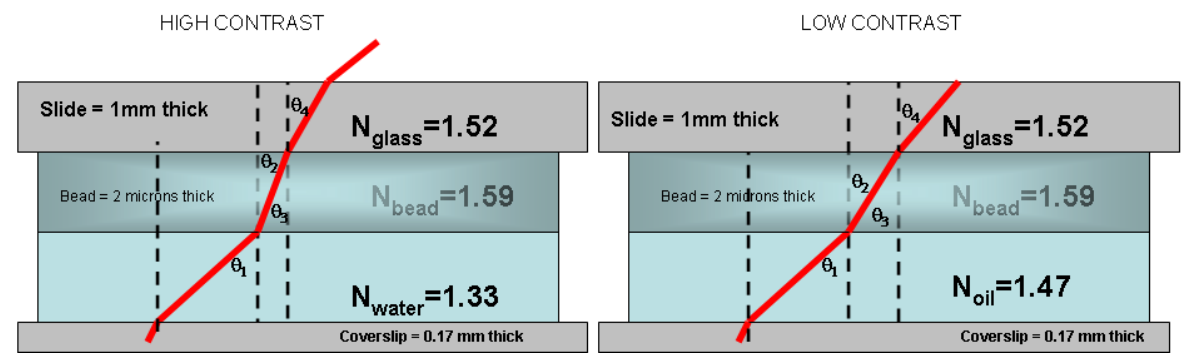

Figure 18: Dry beads onto two slides. Make one slide with water and one with oil to test the response of the QPD as the beam is scanned across the bead and diffracted. Treating the bead like a slab of polystyrene, ray goes through two refractions. Say that $\theta_{1}=15$ degrees. Both cases give the same resulting angles, but because $\theta_{4}$ is greater in the water case, the displacement of the beam is further from the entrance point than in the oil case. 


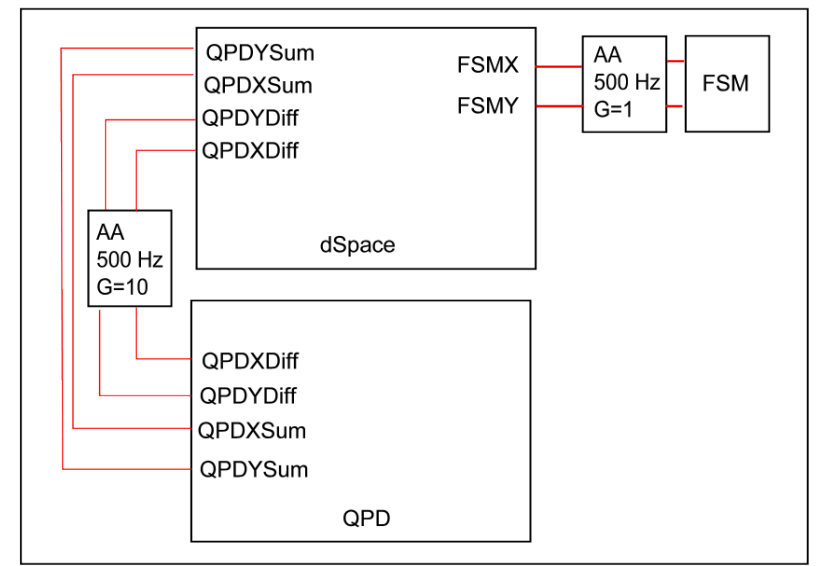

Figure 19: System with four AA's to make clean measurements.

The QPD voltages also depend on where the beam is focused in the $\mathrm{Z}$ direction, into the sample. As the focus is moved from the shallowest region of the bead, through the bead to the deepest, the bead apparently grows and shifts according to the QPD voltages. This means that the measurements taken in the experiment will also depend on focus depth.

The QPD must be zeroed by checking what position will give a "no-beam" response. This is not zero volts. It is more like $-1.1 \mathrm{~V}$ for one channel and $-1.6 \mathrm{~V}$ for the other channel. This measurement will enable the determination of velocity that the bead is dragged during calibration experiments.

The signals of QPDx and FSMx should have high coherence and the signals of QPDy and FSMy should have high coherence. This can be measured by finding the transfer function between the FSM input and the QPD output. It is important that in a scan of the bead, the beam is only scanned within the linear portion of the QPD's response curve. The beam should stay within about a half micron from the center of the bead. Non-linearities would show up as extra noise. Figure 19 shows the system that was used to make the coherence measurements etc. 

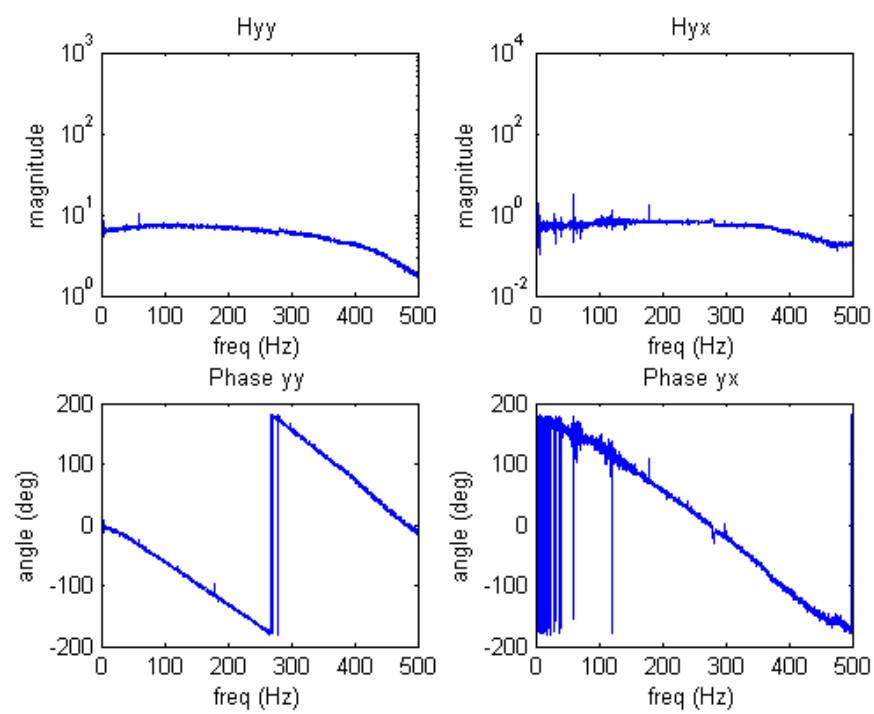

Figure 20: FSM to QPD transfer function (in linear region) for $\mathrm{y}$ to $\mathrm{y}$ and $\mathrm{y}$ to $\mathrm{x}$ (cross channel). The trailing off of the data around $400 \mathrm{~Hz}$, is due to the $500 \mathrm{~Hz}$ cutoff of the AA filter.

The coherence of an input/output system has to do with how related the output is to the input. A coherence of one says that a certain input after a certain initial condition produces the same response every time at the output. It is desirable for this set-up to have a coherence of one in all four cases, FSMy to QPDy, etc. It is shown in Figures 22 and 23 that the coherence is near one for all pertinent frequencies in the two corresponding channels (FSMy to QPDy and FSMx to QPDx). The cross channels are not as important, but they are also around one for most of the spectrum.

In the linear region of the QPD response (as shown in Figure 16), the FSM to QPD transfer function should be flat. It can be seen in Figure 19 that one AA filter applies a gain of one of the signals coming from the dSpace board while the other AA filter applies a gain of 10 to the signal coming from the QPD to increase the dynamic range of measurement. The trailing off of the data around $400 \mathrm{~Hz}$ in Figures 20 and 21 is due to the $500 \mathrm{~Hz}$ cutoff of the AA filter. The fact that the magnitude of the gain is not one is due to the fact that there are factors of 10 between the input to dSpace and the output. 

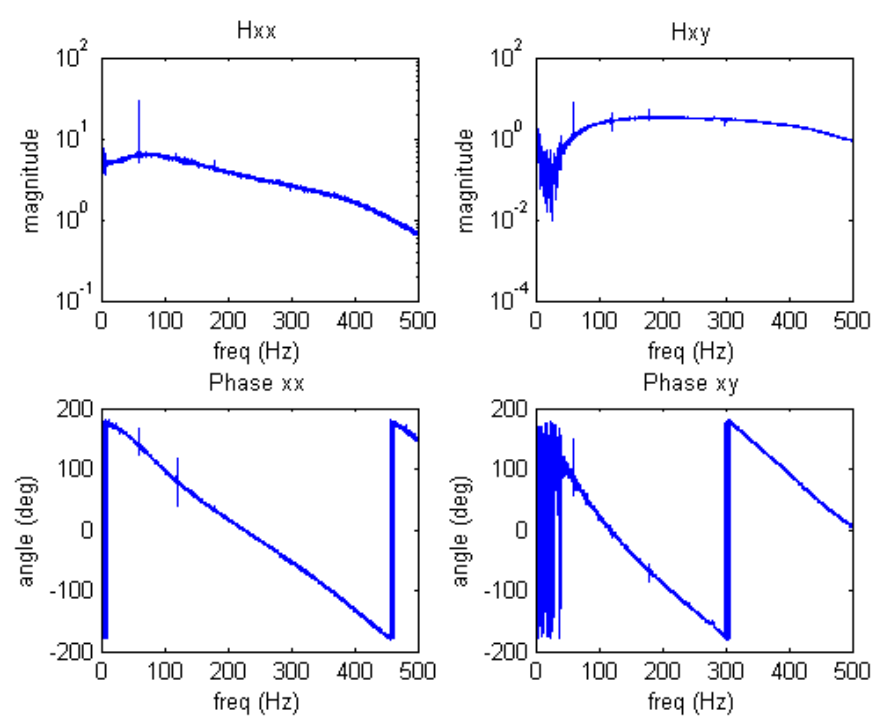

Figure 21: FSM to QPD transfer function (in linear region) for $\mathrm{x}$ to $\mathrm{x}$ and $\mathrm{x}$ to $\mathrm{y}$ (cross channel). The trailing off of the data around $400 \mathrm{~Hz}$, is due to the $500 \mathrm{~Hz}$ cutoff of the AA filter.

The non-zero slope of the phase shows that the "group delay" of this set-up is not constant. This means that there is dispersion (certain frequencies are delayed more than others).

\subsubsection{Trap Stiffness}

There are several ways to find the trap stiffness, which is the effective "spring constant" of the potential well created by the interaction between the laser and the bead. They include the escape force method, the drag force method, the momentum transfer method, the power spectrum method, the step response method, and the equipartition method [43]. Each one requires different knowledge about the particle and its surroundings. This experiment will use the method that requires the least information about the environment of the experiment, the equipartition method. 


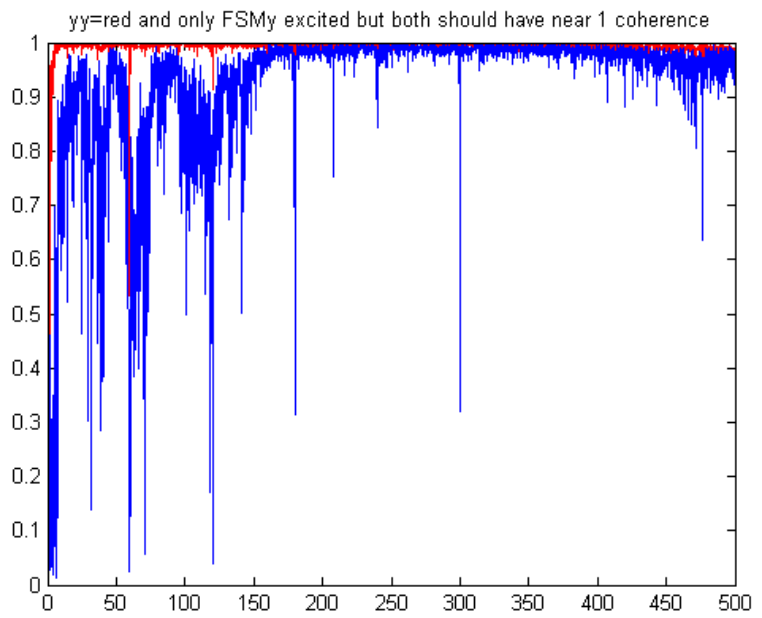

Figure 22: Coherence of FSM to QPD for $\mathrm{y}$ to $\mathrm{y}$ and $\mathrm{y}$ to $\mathrm{x}$. The coherence is near one for all pertinent frequencies in the two corresponding channels (FSMy to QPDy and FSMx to QPDx). The cross channels are not as important, but they are also around one for most of the spectrum.

The related power spectrum method will be used to find the effective viscosity of the interface, a quantity that is unknown, and uncertain in the literature. The power spectrum from the $x$ and $y$ channels of the QPD need to be approximately equal to prove that there is a circular beam, instead of an elliptical beam.

2.5.3.1 Equipartition Method The stiffness of the trap can be found by trapping a bead with no input to the FSM, and measuring the Brownian motion of the bead in a stationary beam with the QPD.

The equipartition method can be used to find the stiffness of the trap by measuring the mean square displacement of the bead in a stationary trap due to Brownian disturbances, using the volts/nanometer transfer function from scanning the dried bead.

Importantly, the viscous drag coefficient is not needed for the equipartition method because the viscous drag of the bead on the interface is unknown [43]. The quadrant photodiode 


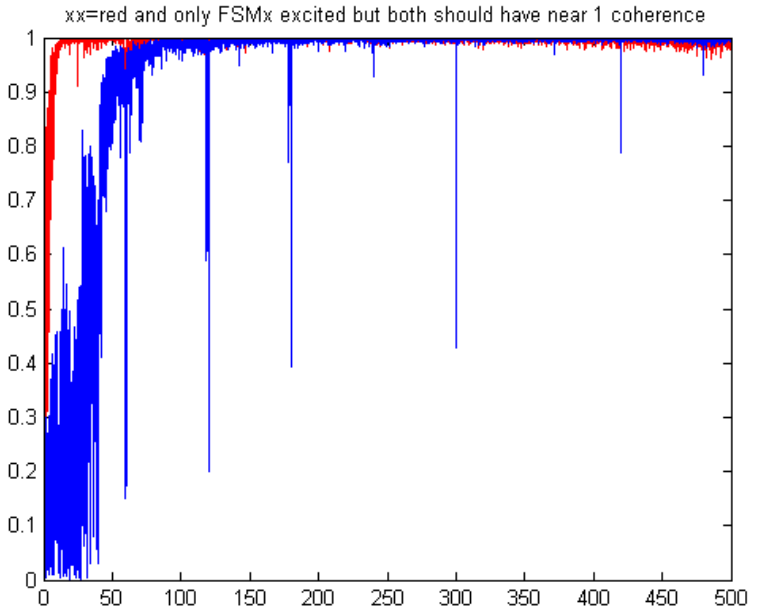

Figure 23: Coherence of FSM to QPD for $\mathrm{x}$ to $\mathrm{x}$ and $\mathrm{x}$ to $\mathrm{y}$. The coherence is near one for all pertinent frequencies in the two corresponding channels (FSMy to QPDy and FSMx to QPDx). The cross channels are not as important, but they are also around one for most of the spectrum.

(QPD) has a high bandwidth and can record all the minute movements of the bead within the trap to obtain the mean square displacement. The trap stiffness is found using the equation:

$$
\frac{1}{2} k_{B} T=\frac{1}{2} k_{\text {trap }}\left\langle x^{2}\right\rangle
$$

First a bead is trapped on the interface and a frequency spectrum of the QPD data is taken at $1 \mathrm{kHz}$, with an AA filter at $3 \mathrm{kHz}$. The trap will be assumed stationary here, so the data collected at the QPD can be used to determine the distance the bead is pushed out of the trap by Brownian motion. An example set of data can be seen in Figure 24.

After removing the bias from the data, it is found that the mean square displacement of the bead is

$$
<x^{2}>=1.4 \cdot 10^{5} \mathrm{~nm}^{2}
$$




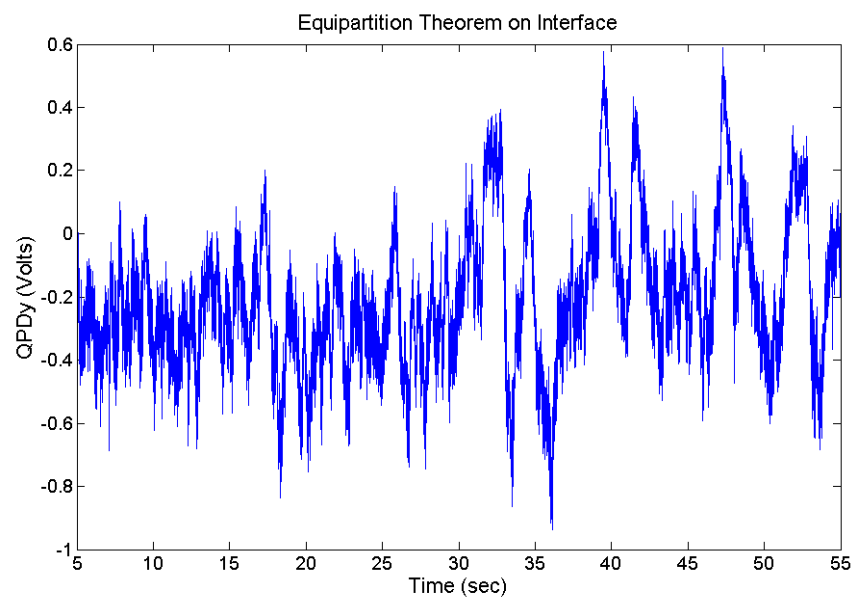

Figure 24: Random movements of bead on the interface.

and from the value of $k_{B} T$,

$$
\frac{4 \mathrm{pN} \cdot \mathrm{nm}}{1.4 \cdot 10^{5} \mathrm{~nm}^{2}}=2.86 \cdot 10^{-5} \frac{\mathrm{pN}}{\mathrm{nm}}
$$

which is the trap stiffness on the interface, $k_{\text {trap }}$. Using this trap stiffness, the Frequency Spectrum Method can be used to find the other unknown of the system, the effective viscosity of the interface.

2.5.3.2 Frequency Spectrum Method The frequency spectrum method uses the fact that the trap-bead system has two first order poles. The pole corresponding to the stiffness on the hydrodynamic drag, $\frac{k}{c}$, is much lower than the pole corresponding to the hydrodynamic drag on the mass of the bead, $\frac{c}{m}$ (see the equation describing the system above). In the following equations, the hydrodynamic drag will be represented with $\gamma$ instead of $c$. The equation relating the cutoff frequency to the stiffness follows:

$$
k_{\text {trap }}=\Omega \gamma
$$

where $\Omega$ is the cutoff frequency and $\gamma$ is the hydrodynamic drag. The hydrodynamic drag is:

$$
\gamma=6 \pi r \mu_{\text {medium }}
$$




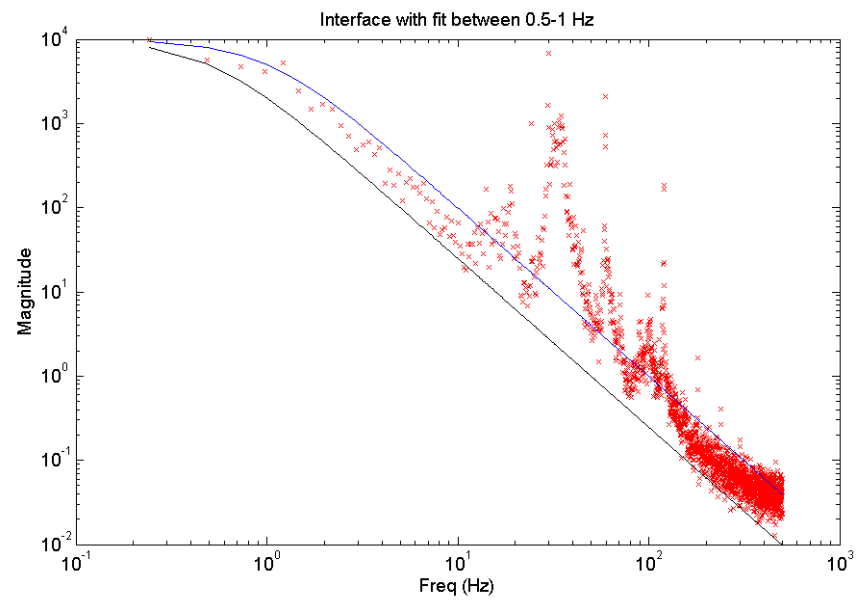

Figure 25: Power spectrum of equipartition data with fits of 0.5 and $1 \mathrm{~Hz}$.

where $r$ is the radius of the spherical particle and $\mu_{\text {medium }}$ is the viscosity of the medium. Using the stiffness derived from the equipartition method, it is possible to find the effective viscosity:

$$
\begin{aligned}
& \pi\left[\frac{\mathrm{rad}}{\mathrm{s}}\right]=\frac{2.86 \cdot 10^{-5}\left[\frac{\mathrm{pN}}{\mathrm{nm}}\right]}{6 \pi \mu_{\text {effective }} \cdot 10^{-6}[\mathrm{~m}]} \\
& \mu_{\text {effective }}=4.83 \cdot 10^{-} 4[\mathrm{~Pa} \cdot \mathrm{s}]
\end{aligned}
$$

The effective viscosity of the interface is found to be about half that of water alone. This might have to do with the fact that the interface pulls the particle in the direction of its motion more than it holds the particle back against its motion. 


\subsection{COLLOIDAL INTERACTION ENERGIES AT AN INTERFACE}

There are two aspects to the behavior of the microspheres at the interface. First there are forces that keep the particles on the interface. Second there are forces between the particles. The experiment will measure the forces between the particles, but explanation of the forces keeping the particles on the interface is also important for completeness. The measurement and understanding of these forces is important in the prediction of the stability of colloids. For example if a certain diameter particle is introduced into a mixture of oil and water, the properties of the particle can cause the mixture of the two immiscible liquids to form into a stable colloid of water with small particle bounded "bubbles" of oil, or vice versa. The properties of this two-part liquid would be different depending on whether the oil or water was the bulk phase.

In this research, there is a well with water on the bottom half and oil on the top half. Polystyrene beads are introduced from above. The beads are heavier than both oil and water and will sink. As the beads sink through the interface between the oil and water, many adhere to the interface and stay there.

There are three main forces causing the particles to interact: 1. van der Waals forces

cause attraction. 2. Capillary forces cause attraction. 3. Like charges cause electrostatic repulsion (Coulomb and/or dipole-dipole) [14]. Only their sum can be measured. 


\subsection{VAN DER WAALS FORCES}

The van der Waals force has to do with induced dipole interaction. The electron cloud of one atom or molecule is constantly changing its instantaneous configuration. This force applies to both polar and non-polar molecules. The overall interaction is always attractive for like materials no matter what the intervening medium [31].

The van der Waals force is characterized by a number called the Hamaker coefficient. Every material has its own value, which also depends on the materials with which it is in contact. For example, polystyrene has a Hamaker coefficient, $A_{\text {Ham }}$ (in the limit of small separation) of $13 \mathrm{zJ}$ across water and $79 \mathrm{zJ}$ across a vacuum [31]. There are instances where the interaction energy between two polystyrene spheres can be significant compared to kT:

$$
E_{i}=\frac{-A_{\text {Ham }}}{12} \frac{R}{l}
$$

where $E_{i}$ is the interaction energy, $A_{H a m}$ is the Hamaker coefficient, $R$ is the radius of the particles, and $l$ is their separation [31]. This equation applies with $l \ll R$. A situation where $E_{i}$ would be greater than $\mathrm{kT}$ would be if $A_{H a m}>k T$ and $l<R / 12$. In water, polystyrene's $A_{\text {Ham }}$ is about $3 \mathrm{kT}$. For the 1 micrometer radius bead's used in this experiment, they would have to be less than $300 \mathrm{~nm}$ apart for van der Waals forces to dominate. The beads are normally much further away than this, but they are bound to come close enough every once in a while and this helps explain their aggregation over time. The DLVO theory treats the van der Waals and electrostatic forces as though they are separable even though they are not [31].

The interaction force (derivative of the energy equation above) between two spheres (very close together) varies with $1 / l^{2}$ where $l$ is the separation (between the surfaces of the particles) [31].

$$
F=-\frac{d E}{d l}=\frac{A_{H a m} R}{12 l^{2}}
$$




\subsection{CAPILLARY FORCES AND SURFACE TENSION}

Capillary forces are caused by surface tension. The capillary forces are proportional to the amount the beads deform the interface. The beads in this experiment are so small that the interface is effectively flat. There are surface tensions between the particle and oil, between the particle and water, and between the oil and water, which act upon all particles at the interface [35]. Where the bead sits within the interface, i.e. more surface area contacting the water or the oil has to do with the beads hydrophobicity. The weight of the bead is about $66 \mathrm{fN}$ while the force due to surface tension between water and polystyrene is $63 \mathrm{nN}$, about 6 orders of magnitude. This is assuming the surface tension between water and polystyrene is about $100 \mu \mathrm{N} / \mathrm{cm}$.

Force due to surface tension $=$ surface tension $\cdot$ circumference of bead

Force due to surface tension $=100 \cdot 10^{-6}\left[\frac{\mathrm{N}}{\mathrm{cm}}\right] \cdot 2 \pi\left(10^{-4}[\mathrm{~cm}]\right)=6.28 \cdot 10^{-8} \mathrm{~N}$

Table 5: Densities of Materials in Experiment in grams per cubic centimeter

\begin{tabular}{lll} 
Water & Mineral Oil & Microsphere \\
\hline 1.0 & 0.9 & 1.06 \\
\hline
\end{tabular}

The fact that any microspheres are adsorbed to the interface shows that their presence lowers the interfacial surface tension between the oil and water, or the tension between oil and microsphere, and water and microsphere[10]. The density of each material should also be noted, in Table 5. The microspheres sink through the oil and eventually end up on the bottom of the well, but some do adhere to the interface. The contact angle is related to the three interfacial tensions shown in Fig 26 by the Young's Equation:

$$
\cos \theta=\frac{\gamma_{p o}-\gamma_{p w}}{\gamma_{o w}}
$$

where $\theta$ is the contact angle [8]. 
There can be an accounting of the surface energy changes. As the microspheres are sinking, there is an interface between the oil and water with area, $A_{o w}$, and there are interfaces between the polystyrene microspheres and the oil with area $A_{p o}$. When the microsphere adsorbs to the interface, a change occurs. $A_{o w}$ decreases, $A_{p o}$ decreases, and an interface between microsphere and water is formed with area, $A_{p w}$.

The surface tension between oil and water is $49 \mathrm{mN} / \mathrm{m}$ [27].

The energy associated with gravity's effect on the microsphere is:

$$
E_{g}=\frac{4}{3} \pi R^{4} \rho g \approx 4.4 \cdot 10^{-20} J
$$

where $R=10^{-6} \mathrm{~m}$ and $\rho=1600 \mathrm{~kg} / \mathrm{m}^{3}$.

Thermal energy at room temperature is:

$$
k_{B} T \approx 4.1 \cdot 10^{-21} \mathrm{~J}
$$

or about 4 zeptojoules (zJ) where the Boltzmann constant $k=1.38 \cdot 10^{-23} \mathrm{~J} / \mathrm{K}$.

It can be shown that the forces experienced by the microspheres on the interface from the optical tweezers will not be strong enough to remove them from the interface. Thus showing that only 2 dimensional movement will occur assuming the interface is flat with respect to the objective. The contact angles between the microsphere, and the oil, and the microsphere and water, are difficult to measure. They would be necessary to know in order to determine the forces experienced by the microspheres due to the interface using other methods, but the use of optical tweezers eliminates the need to know these angles.

\subsection{ELECTROSTATIC FORCES}

The microspheres have some charge. They sit in a solution for weeks at a time, and get shaken every once in a while. It is possible that they were functionalized with some ionic substance that would give them a permanent charge. Also, they could have picked up charge from the solvent in which they sit. In this section some possible interaction energy pair 


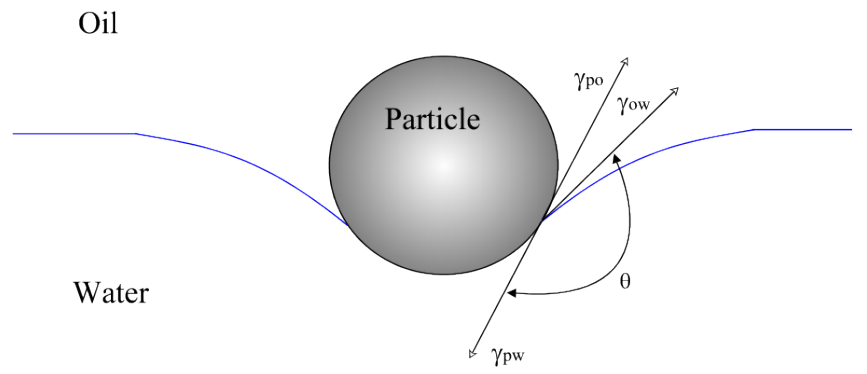

Figure 26: Diagram of surface forces acting on bead, independent of other particles.

potentials will be discussed. The Coulomb potential describes the interaction between two charged objects. The Coulomb potential is:

$$
U_{\text {Coulomb }}(r)=k \frac{q_{1} q_{2}}{r} .
$$

where $q_{1}$ and $q_{2}$ are the charges, $\mathrm{k}$ is some constant, and $\mathrm{r}$ is the distance between the charges. In the case of this experiment, the microspheres will be modeled as point charges with a charge that is the sum of all the surface charges on the bead. All the r's in the potential equations refer to the distance between point charges. The Lennard-Jones potential applies to neutrally charged objects like water molecules. The Lennard-Jones potential is:

$$
U_{\text {Lennard-Jones }}(r)=4 \epsilon\left[\left(\frac{\sigma}{r}\right)^{12}-\left(\frac{\sigma}{r}\right)^{6}\right]
$$

where $\sigma$ is a parameter in units of meters and $\epsilon$ is in units of $\mathrm{kJ} / \mathrm{mol}$. The Yukawa potential is as follows:

$$
U_{\text {Yukawa }}(r)=-g^{2} \frac{e^{-m r}}{r} .
$$


where $g$ is the coupling constant from quantum electrodynamics, and $m$ is the mass. The pair potential of two colloidal particles is given by the "electrostatic part of the DLVO theory" as follows:

$$
U_{D L V O}(r)=\frac{Z_{*}^{2} e^{2}}{\epsilon}\left[\frac{e^{\kappa \sigma}}{1+\kappa \sigma}\right]^{2} \frac{e^{-\kappa r}}{r}
$$

where $Z_{*}$ is the effective charge of a sphere with radius (assuming both microspheres have the same charge), $\sigma, \kappa^{-1}$ is the Debye-Huckel screening length, and $\epsilon$ is the permittivity of the liquid [12]. This has the same form as the Yukawa theory for a screened Coulomb interaction. The Debye-Huckel screening length is on the order of 10s to 100s of nanometers.

On the interface, the microspheres arrange themselves into a hexagonal lattice. The lattice can be seen in bands across the surface, not actually spreading uniformly across the whole interface. There are multiple lattice constants in the lattice. The edges of the bands have constants near 14.6 micrometers, while the central regions have a constant near 8.8 micrometers, as can be seen in figures 27 and 28 .

In my experiment, there are more forces at work, than just the Coulomb force, but in general, the term used is 'pair potential'. By comparing the shape of the data with these theories, the correct form of the potential can be chosen. The force due to two charges as mentioned in the above section, is inversely proportional to the distance between the two charges.

The dipole-dipole interaction potential between the particles varies with $1 / r^{3}$ if the particle separation is greater than the particle diameter [24]. This would cause a force varying with $1 / r^{4}$. This goes against what all four proposed potentials say.

When the slope of potential is positive, there is attraction between the two particles. The Coulomb potential is repulsive (for particles of like charge) at all separations and gains strength at small separations. The Lennard-Jones potential on the other hand can be attractive at large separations, and becomes very repulsive at smaller separations The Yukawa potential is similar in shape to the Coulomb potential, but is different in magnitude because it accounts for shielding of charges. 


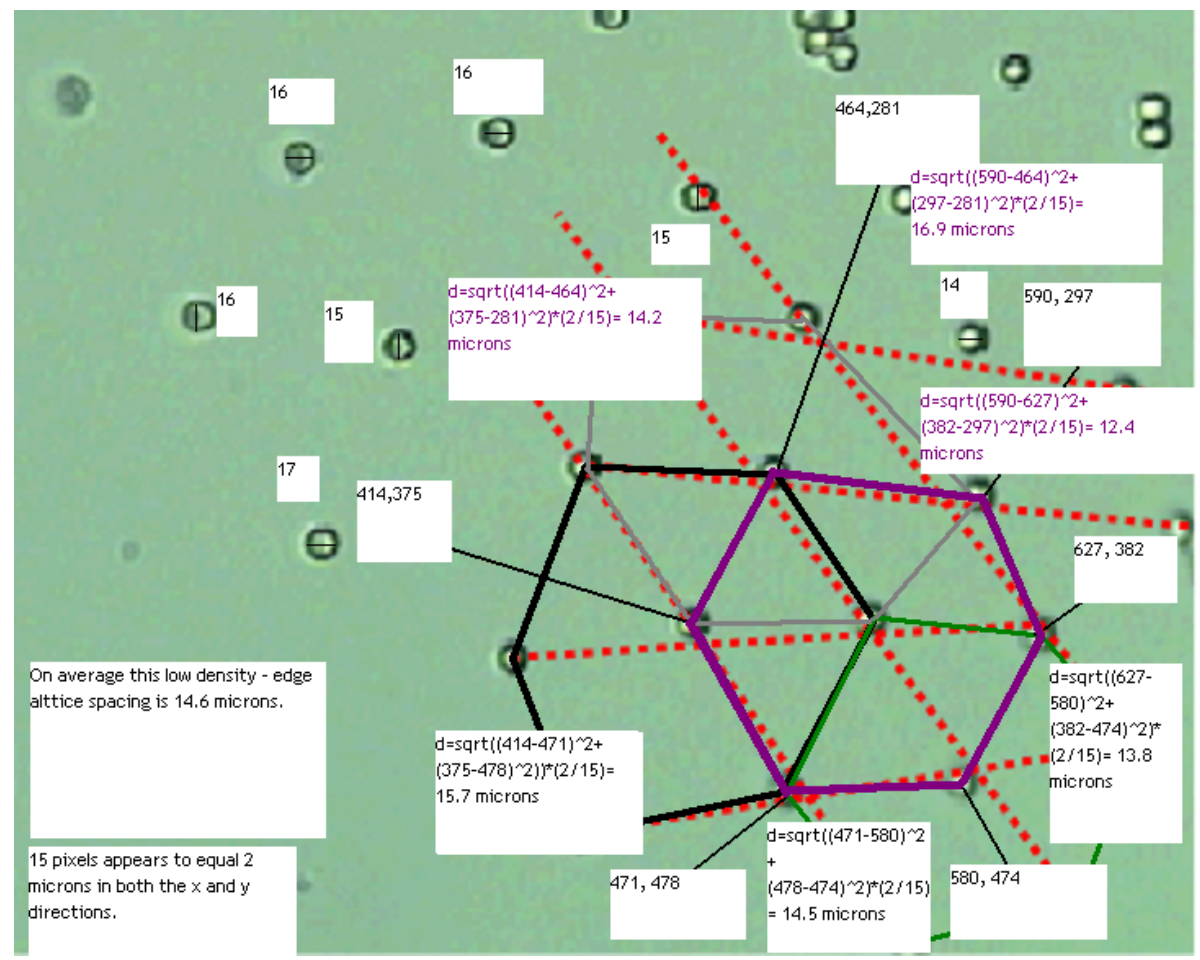

Figure 27: Lattice spacing on edge of a lattice. 


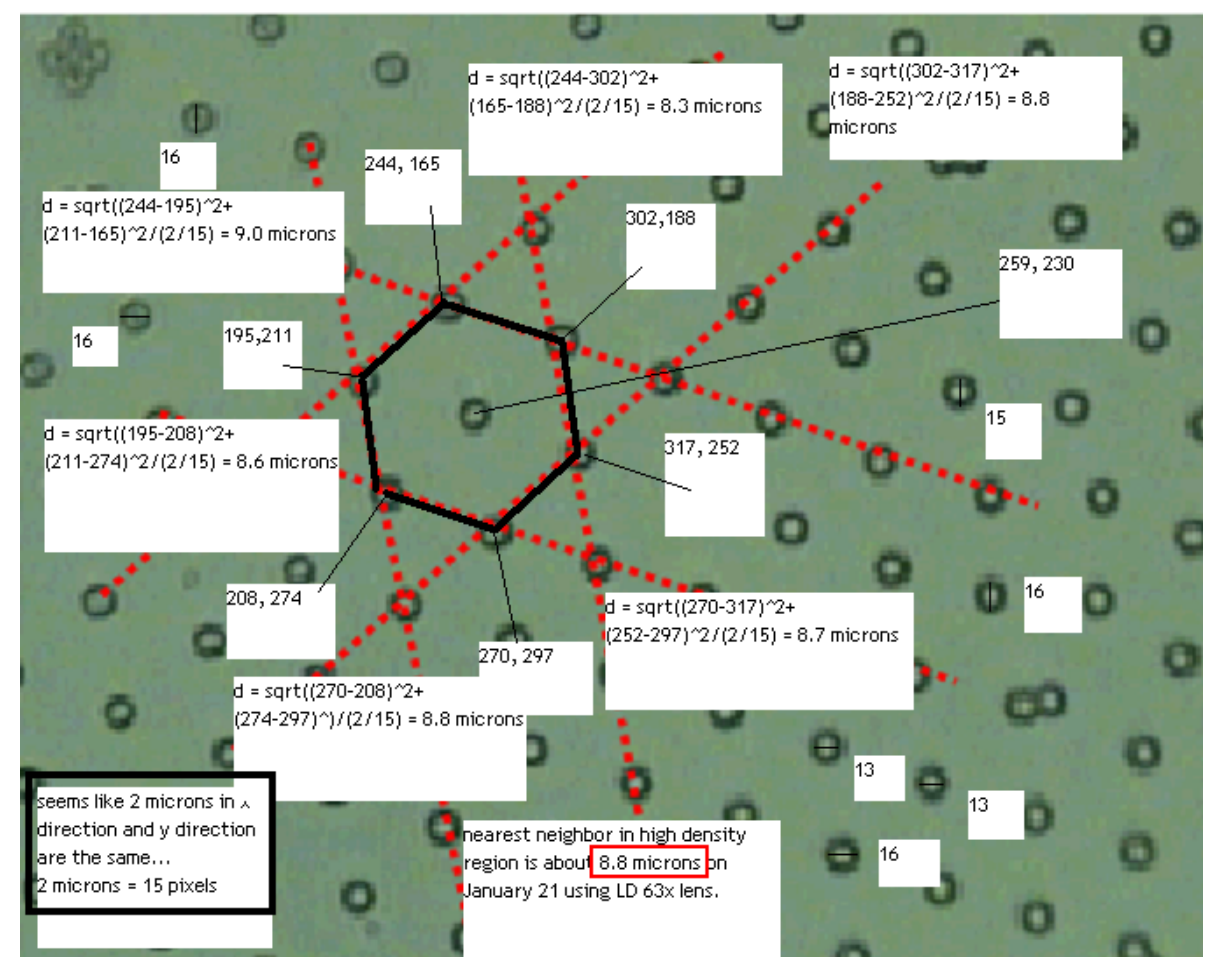

Figure 28: Lattice spacing in center of band. 
Because water is polar the charges around the water-wetted area of the microsphere can move toward the surface, creating a local dipole. The effect of this dipole on the surrounding microspheres is screened by the free charges in the water, but because the oil is non-polar, the charges in the oil do not move, and cannot screen the top half of the dipole, therefore, the interaction between the dipoles can occur through the oil.

Another reason that the electric field from the charges on the microspheres is more significant through the oil phase is that the magnitude of an external electric field is reduced inside any material with a higher dielectric constant than the surrounding medium. The dielectric constants of water, mineral oil, and air are shown in Table 6.

Table 6: Dielectric Constants of Materials in Experiment.

\begin{tabular}{llll} 
Distilled Water & Mineral Oil & Air & Polystyrene \\
\hline 77 & $2-3$ & 1.0 & 2.55 \\
\hline
\end{tabular}

Figure 29 shows a schematic representation of the screening and repulsion of the dipoles formed by the charges on the surface of the microspheres interacting with the free charges in water.

According to Robinson and Earnshaw, the "dipole" that causes the repulsion between the particles is formed by the counterions that are attached to the sulfate groups on the particle in the non-wetted portion, not the attracted counterions from the water [37]. There is "short range repulsive dipole interactions arising from dissociated bound surface groups on the part of the particle" not in the water [37]. Particles manufactured without surfactant make better, more stable crystalline monolayers [37]. The more hydrophobic the particle is, the less screening it experiences in the water phase, and therefore the stronger the repulsion it has on other particles.

Depending on the charge on the microspheres, the dielectric constants of the two materials, the concentration of electrolyte, and the distance between the microspheres, dipoledipole interactions or Coulomb interactions dominate in the electrostatic portion of the inter-particle forces [17]. The dipole-dipole interactions dominate at long distances. 


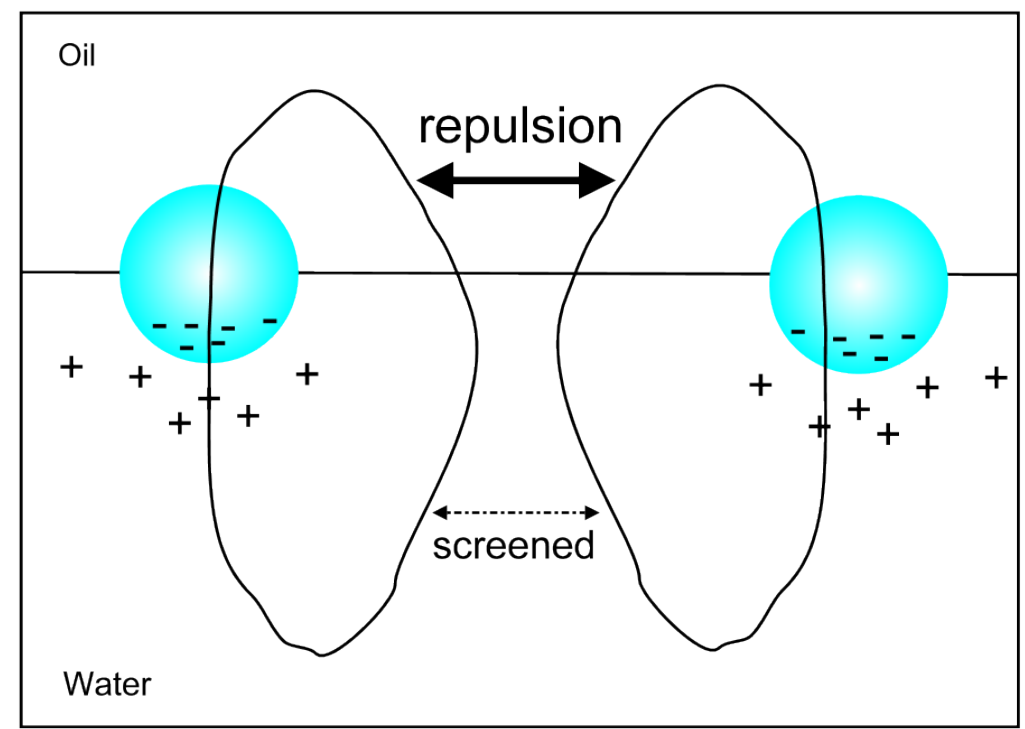

Figure 29: Dipole repulsion acting only through oil. 
It has been shown by several researchers that much higher concentrations of salt (100x) are needed to cause agglomeration of particles on an interface compared to a bulk colloid [37]. This implies that the repulsion is much stronger through the other fluid making the interface, be it air or oil.

The Debye length, $\lambda_{d}$ is defined as the distance from a particle where the electrical potential is equal to the thermal energy. This means that after this length, the electrostatic potential is too low to have a real effect on the motion of surrounding particles. The equation for the Debye length is:

$$
\lambda_{d}=\left(\frac{\epsilon R T}{2 F^{2} z^{2} C}\right)^{1 / 2}
$$

where $\epsilon$ is the relative permittivity, $\mathrm{R}$ is the Universal gas constant, $\mathrm{T}$ is the temperature in Kelvin, F is the Faraday number (the product of Avogodro's number and the charge of an electron), $\mathrm{C}$ is moles per unit volume (concentration of the electrolyte), and $\mathrm{z}$ is the valence of the electrolyte. De-ionized water is used in the experiment, so the electrolyte concentration is very low. The addition of salt to the water would decrease the Debye length. This means for the given temperature, the Debye length is at the maximum value it could be in water. Yet even at this maximum value, it is believed that the majority of the electrostatic force is mediated through the oil.

Dai et al. found that electrostatic repulsion does not completely explain lattice formation. The surface treatments of the microspheres in their experiment included sulfate, carboxylate, and amine. All of these surface treatments add charge to the microsphere, and yet only the sulfate-treated microspheres formed self-assembled lattices [28]. This implies that the chemistry of the surface also has something to do with lattice formation. 


\subsection{EXPERIMENT}

The experiment involves measuring the light scattered from beads that are pushed from centers of optical traps by interaction forces. The polystyrene beads are on an interface between oil and water, and are therefore confined to two dimensions.

\subsection{METHODS}

The interface on which the beads in this experiment will lie, is formed by placing about 12 microliters of water on the bottom of the well with a microsyringe. Then 12 microliters of oil are placed on top of the water with a sharp piece of aluminum. In the amount of time it takes to get the oil, air has already contacted the surface of the water. Surface tension changes dramatically with the addition of impurities, and it is assumed that the effect of impurities is about the same in every sample [10]. The well is $5 \mathrm{~mm}$ in radius and about $1.5 \mathrm{~mm}$ deep. It is made by drilling a hole in a piece of plastic and attaching it to a No. 1 coverslip with nail polish. A model of the well can be seen in Figure 2. The No.1 coverslip is $0.17 \mathrm{~mm}$ thick. In order to help the beads spread across the interface, isopropanol is added to the bead solution. One dilution used in a previous work is 1 part bead solution, 4 parts isopropanol and 5 parts deionized water [40]. This reduces the concentration of beads to a manageable level $\left(\approx 2 \times 10^{7}\right.$ microspheres $\left./ \mathrm{mL}\right)$. The microsyringe is used to place a small droplet of microsphere solution into the oil in the well. The beads sink through the oil, and some get trapped on the interface between the oil and water. The rest sink through into the water layer. 
Polystyrene microspheres self-assemble into hexagonal lattices when they are confined to two dimensions on the oil-water interface. Plots of force versus position will be made. These plots will be compared with the four potentials mentioned above to see which they match most closely with. The theoretical values that would take into account charge on the microspheres, the surface tension of the oil and water, etc. will be absorbed into some general fitting parameters.

Proximity to walls can change viscous forces and create electrostatic forces on the beads[13]. To prevent this it is important that the beads are a few microns from the coverslip. The layer of water provides this needed separation. The reason the water layer can be thick enough, is that the objective has a long working distance.

Note: When the vial of microsphere solution is not shaken, beads will adsorb to the interface, but the hexagonal lattice will be less pronounced or not present at all, and measurements confirm that the beads do not exert the same force on each other in this state even though they still appear to be in a plane, i.e. on the interface.

Note: When the microsyringe is used to inject the microsphere solution into the interface, the oil reacts in such a way that it retreats from the injection site, ripples a little, and then re-covers the open patch of water that was created when it retreated. If this does not happen, it is likely that no beads were deposited on the interface.

Note: The interface can have flow patterns. From the measurements taken, there was no noticeable pattern as to what conditions caused what flow patterns. These flow patterns increase in velocity and change direction depending on whether the laser is on, and which power setting is chosen. This can make trapping two beads to move towards each other very difficult. Even if two beads can be trapped, the flow causes other beads to bump into them disturbing the measurement being taken.

Because the trap is formed by a low-NA lens, radiation pressure causes several things to happen throughout the calibration and interaction force measurements. When dragging beads through the bulk, the bead is pressed against the slide, creating extra drag by Faxen's Law. When dragging beads along the interface, the bead can be pressed further into the oil phase than it would naturally sit. Sometimes beads are kicked up from the bulk water and pressed all the way up to the interface. This interferes with measurements and makes it 
difficult to take long samples. It is unknown whether the laser being used is powerful enough to push one of these "kicked-up" beads into the interface. They can easily be distinguished from beads adsorbed to the interface because they can be moved around much more easily by the trap. Also, as the trap focus is made deeper, the "kicked-up" beads behave similarly to the beads in bulk water as the focus is deepened toward the slide. It can be seen that the particles in this setup are always many particle radii from the well walls and the coverslip surface, so these do not need to be taken into account with drag calculations. The effect of charge shielding by the water can also be studied by comparing the "kick-up" beads with the beads on the interface, and also with the different focus depths.

The stiffness of the trap was measured when the bead is in the middle of a large lattice and also when a bead is pulled from the edge of the lattice and measured alone. The stiffness is the same in both cases. The optical properties of the trap are obviously the same in both cases, but this also means that the "effective viscosity" of the interface is the same whether the bead is in a lattice or not. 


\subsection{RESULTS AND DISCUSSION}

All measurements made at the interface assume a stationary interface, though in many cases, the interface seems to be "flowing" due to heating by the laser. By taking averaged data it should be possible to remove any bias from a steady movement of the beads.

For the interaction force measurement the FSM trapped bead is dragged slowly towards and away from another bead trapped with the stationary beam. Only one axis is necessary because the force is radially symmetric. Because there are diffraction bands, in the vertical direction, there will be less distortion of the measurement dragging the bead parallel to the bands (i.e. along the y-axis of the viewing screen).

There is a diffraction pattern likely from the dichroic mirrors that is superimposed on the image of the interface when viewed through the microscope. As the laser is rastered across the screen, it passes through vertical stripes that blur and reduce its brightness. There are about 100 vertical stripes across the width of the microscope field of view. The QPD signal seems to vary in correspondence with these stripes, changing in intensity. The width of the stripes (3-4 microns) is about the period of the fluctuations in the data in the $\mathrm{x}$ direction. One way to remove these fluctuations is to measure the total intensity on the QPD simultaneously, and normalize all the data to this overall intensity. There is only $y$ direction data shown here, making normalization unnecessary.

Note: It is unnecessary to consider all the beads that are floating in the water below the interface and the oil above the interface because only the bead in the focus scatters

light strongly. Any other beads that are in line with the beam and condenser would have negligible scattering.

According to Dai et al. "the diffusion of microspheres on an oil-water interface is dominated by the characteristics of the oil phase" [28]. This means that it may be valid to assume 


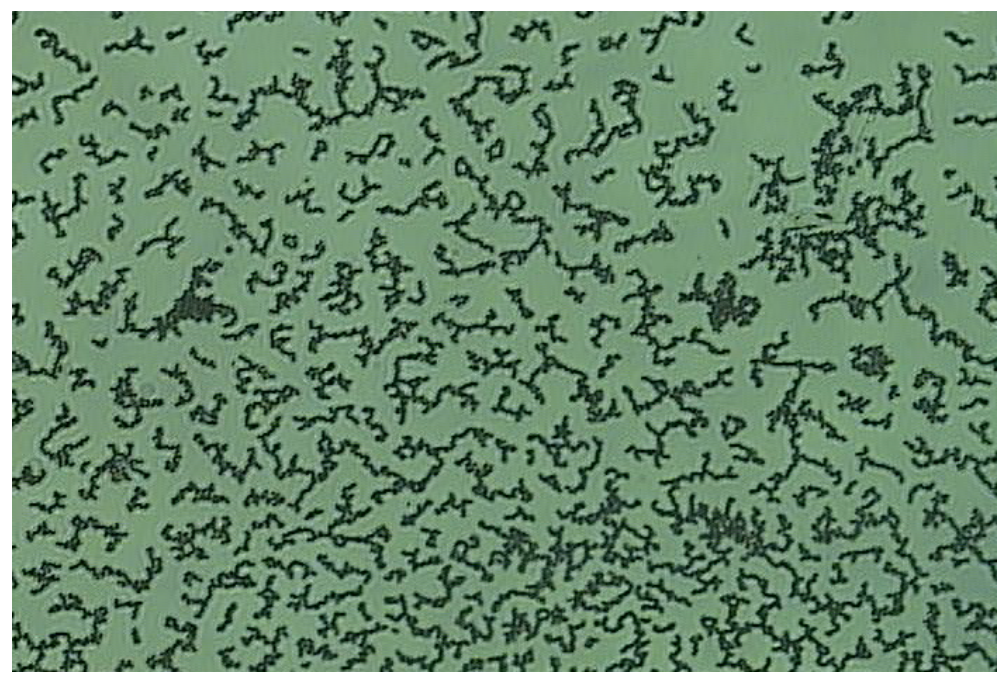

Figure 30: Sample with bunches of beads after 18 hrs.

the viscosity of the oil as the "effective viscosity" of the interface. Their determination was based on the fact that the diffusion constant found using the Stokes-Einstein relationship (described in the Future Work section) matched the viscosity of the oil much more closely than that of water. The other main factor in determining the dynamics of a microsphere at the interface is its wettability [28]. Diffusion is faster for particles that are more hydrophilic because the extra viscosity of the oil does not hold them back as much. A value for the effective viscosity of the interface was already found during calibration, and will be used in the interpretation of the experimental data.

There is change that occurs over time with the sample. If it is allowed to sit for 18 hours, the beads slowly agglomerate into bunches. Figure 30 shows the bunches of beads to compare with the arrays in a fresh sample. Because the water is completely covered, it can be assumed that the ionic concentration of the water is not changing with time. It is possible that the oil is collecting materials from the air which ionize and help shield the repulsion of the particles through the oil phase allowing attractive van der Waals forces to be large enough to pull particles together [37]. 
It is helpful that the diameter of the particles is known because that is the main source of error in Bishop et al, contributing an error of $5 \%$ in their measurements of viscosity in a liquid [2].

\section{$5.1 \quad$ ASSUMPTIONS}

Many assumptions must be made to find actual values for the forces:

The bead could potentially be rolling, again changing the concept of "viscosity".

The interface is not flowing or assume the flow that occurs during each experiment is significant, so that it must be averaged out by several measurements.

The cutoff frequency of the trap while holding a bead still on the interface was measured as $1 \mathrm{~Hz}$, but is probably not correct, it is probably even lower.

\subsection{GAUSSIAN TRAP PAIR POTENTIAL MEASUREMENTS}

Measurement set stats: "Crossforce4" data was taken with two beadsoutside the lattice. FSM beam $=80 \mathrm{~mW}$, Stationary beam $=100 \mathrm{~mW}$. "Crossforce6" is assumed to have the same powers as "Crossforce4" but was taken with the beads inside the lattice. Table 7 shows the conversion of QPD volts to bead displacement out of the trap, used in the interpretation of the experimental data.

Table 7: Conversions in Force Calculation.

\begin{tabular}{ll}
\hline QPDvpmY & $1.6 \mathrm{QPD}$ volts per micron for $\mathrm{Y}$ \\
\hline QPDvpmX & $2.1 \mathrm{QPD}$ volts per micron for $\mathrm{X}$ \\
\hline
\end{tabular}

The FSM beam had a power of $80 \mathrm{~mW}$ while the stationary beam had a power of 100 $\mathrm{mW}$. This means that the stationary bead was moving approximately as much as the moving 


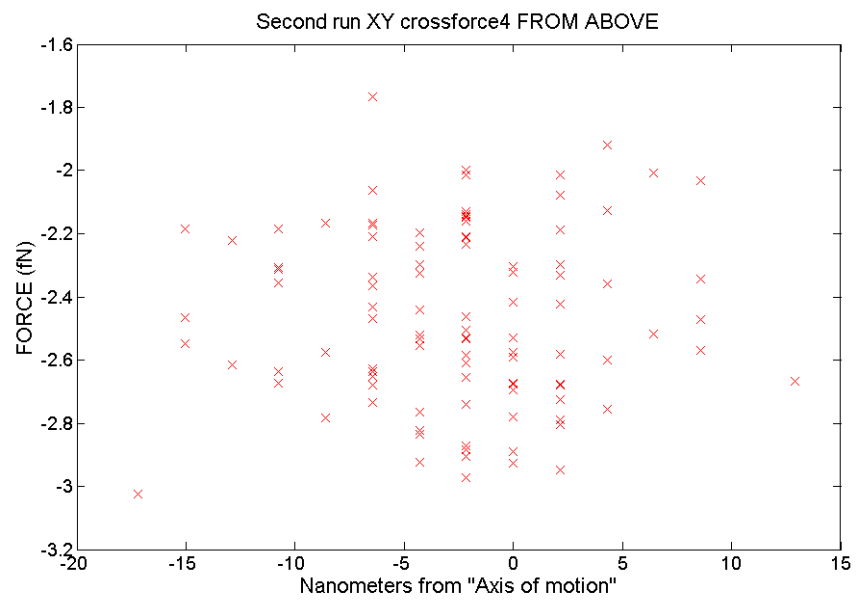

Figure 31: Force on bead with respect to distance from each other in direction perpendicular to movement.

bead. Because the traps are not coupled, the force measured at the FSM trap, is equal to the force that would be measured at the stationary trap. It is only necessary to make the one measurement.

The QPD is used to determine the position of the bead with respect to the beam. The QPD volts vs. FSM raster data is used to find the QPD response of the moving beam with a stationary beam. The dynamic range of the QPD response is proportional to the power in the raster beam. Therefore, the $30 \mathrm{~mW}$ raster beam can be used to determine the meaning of QPD data found with an $80 \mathrm{~mW}$ trapping beam. The calibration data from the raster must be scaled by $80 / 30$ for example.

Figures 31 and 32 show the force measured in the direction perpendicular to the motion of the FSM bead toward the stationary bead. This channel should show zero force, but does not because there are Brownian disturbances acting on the bead in this direction, and there are also random flows on the interface. This information can be used to compare the interfacial "flow" between data sets. 


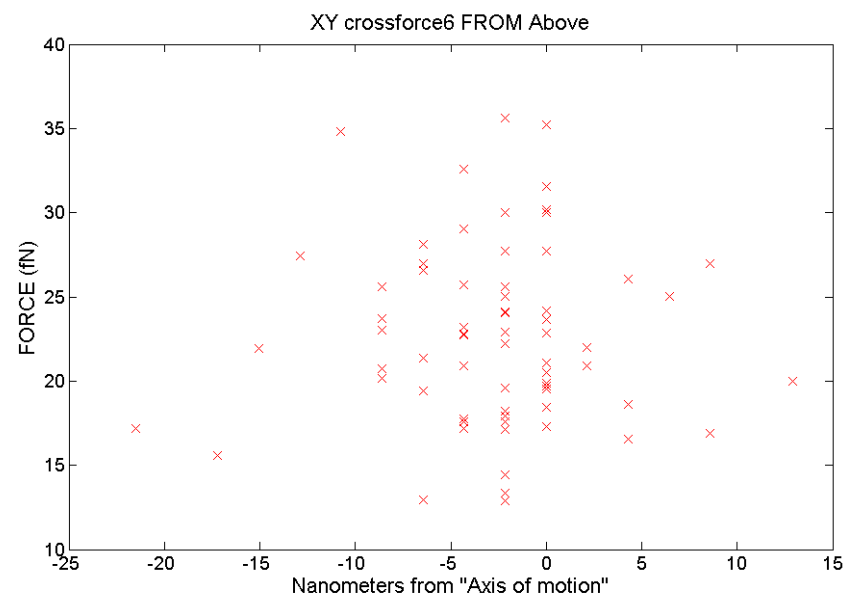

Figure 32: Force on bead with respect to distance from each other in direction perpendicular to movement.

The charges on the surface of the particles can cause them to attract or repel according to the Coulomb force:

$$
F=k \frac{q_{1} q_{2}}{r^{2}} .
$$

where

$$
k=\frac{1}{4 \pi \varepsilon_{0}}
$$

and $\varepsilon_{0}=8.854 \cdot 10^{-12} \mathrm{C}^{2} / \mathrm{Nm}^{2}$. In general the Coulomb force varies as $r^{-2}$ where $\mathrm{r}$, is the center-to-center distance of the microspheres. The dipole-dipole force varies as $r^{-3}$.

Figures 33 and 34 show the force in the direction parallel to the motion of the FSM bead toward the stationary bead. The forces are very small, showing the sensitivity of the measurement method. The fit is a general $r^{-2}$, Coulombic fit. The $\mathrm{r}$-squared values of the fit, are reasonably high.

Figures 35 and 36 show the force in the direction parallel to the motion of the FSM bead toward the stationary bead. The forces are very small, showing the sensitivity of the measurement method. The fit is a general $r^{-3}$, dipole-dipole fit. The r-squared values of the fit, are reasonably high and approximately the same as that for the Coulombic fit. 


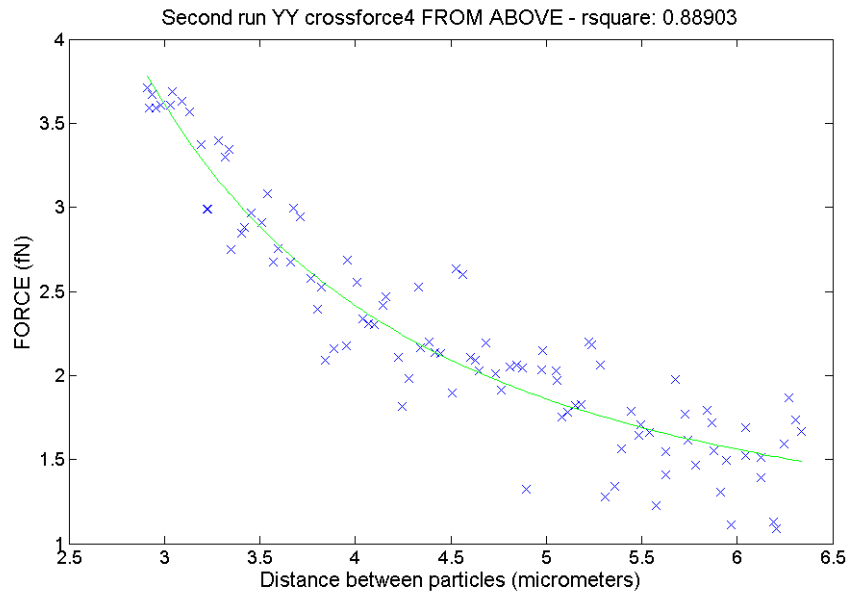

Figure 33: Force on bead with respect to distance from each other in direction parallel to movement with a Coulomb force fit.

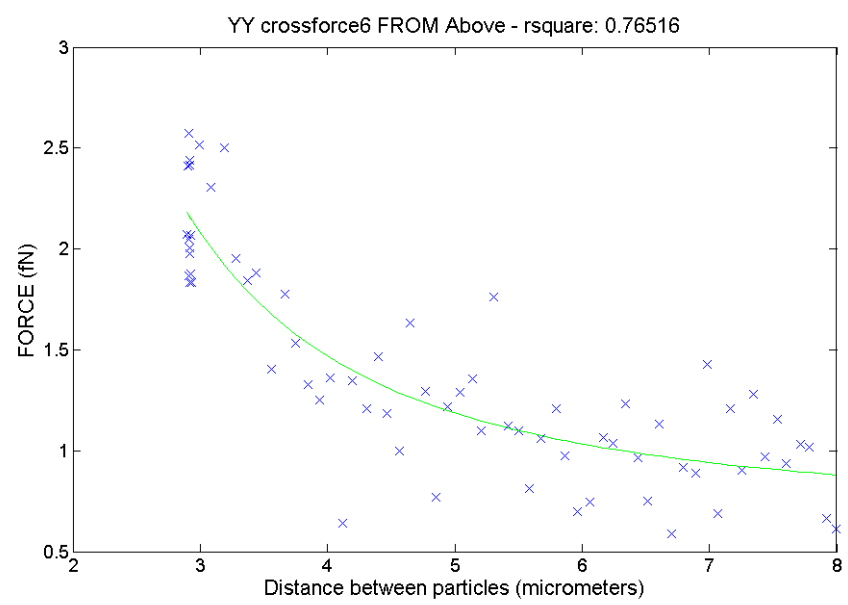

Figure 34: Force on bead with respect to distance from each other in direction parallel to movement with Coulomb force fit. 


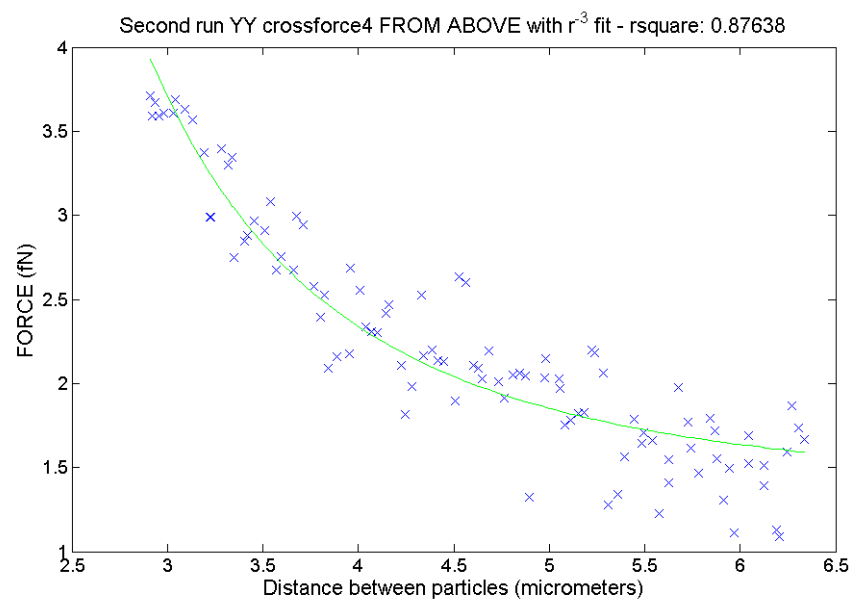

Figure 35: Force on bead with respect to distance from each other in direction parallel to movement with a Dipole- Dipole force fit.

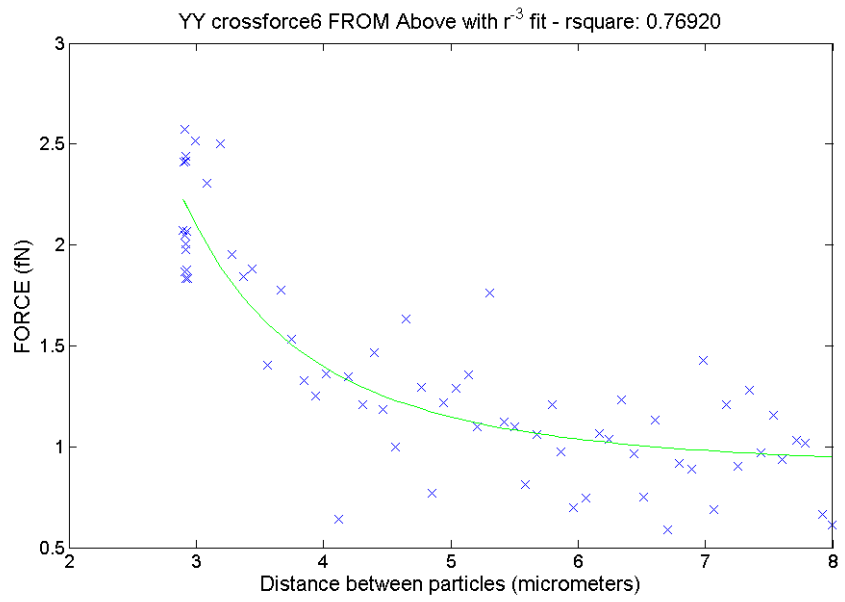

Figure 36: Force on bead with respect to distance from each other in direction parallel to movement with Dipole-Dipole force fit. 


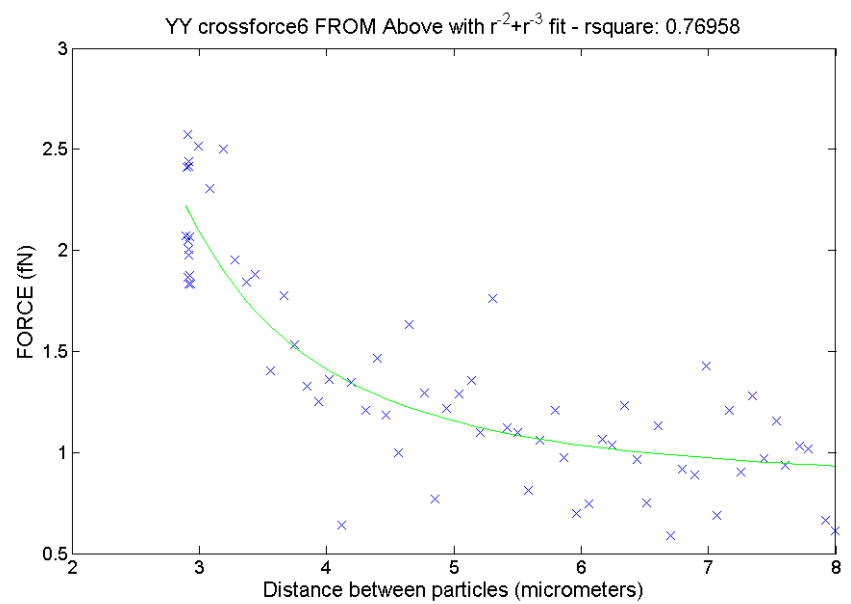

Figure 37: Force on bead with respect to distance from each other in direction parallel to movement with a Coulomb and Dipole-Dipole force combined fit.

Because both fits have approximately the same r-squared value, the fit to an $r^{-4}$ was checked and found to be lower than both Coulombic and dipole-dipole fits. Finally, a combination Coulombic and dipole-dipole fit was performed on the crossforce6 data (see Figure 37) in an effort to maximize the r-squared value, but it stayed about the same.

Table 8: Microsphere Information.

\begin{tabular}{ll}
\hline Company & Interfacial Dynamics Corp. \\
\hline Product & Surfactant-Free White Sulfate Latex \\
\hline Diameter $(\mu \mathrm{m})$ & $2 \mathrm{CV}: 1.8$ percent \\
\hline Solid percentage & 8.1 \\
\hline
\end{tabular}




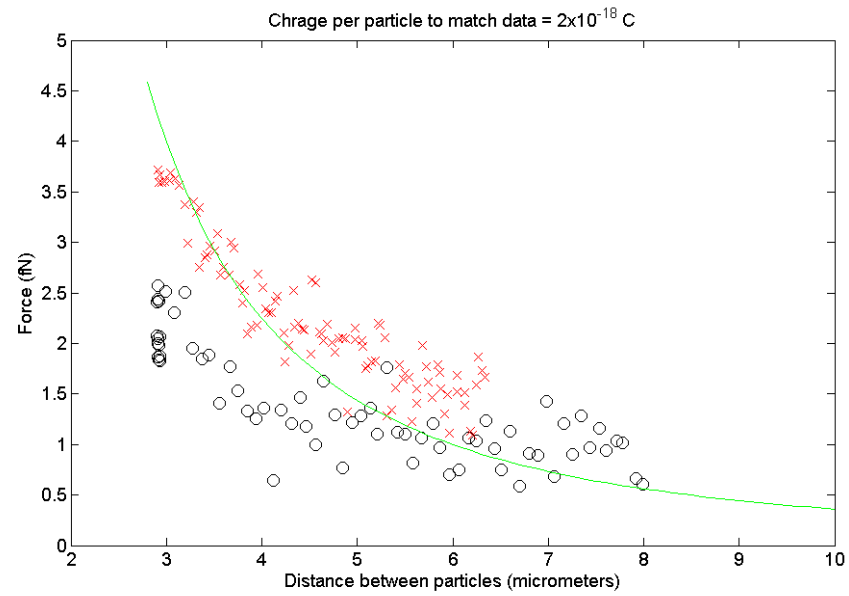

Figure 38: The total surface charge taken as a point charge at the center of two interacting beads necessary to match the experimental data.

The data available about the microspheres is given in Table 8. There is a charge of two electrons on a single sulfate group, and according to the company website, there is one charge group per 2-20 square nanometers. The microsphere has a radius of 1,000 $\mathrm{nm}$ giving it a surface area of $1.256 \cdot 10^{7} \mathrm{~nm}^{2}$. This gives each microsphere a theoretical total surface charge of between $2 \mathrm{pC}$ and $0.2 \mathrm{pC}$. Figure 38 shows the much lower amount of surface charge found to be causing the beads to repel in the experiment. 
The magnitude of the forces expected are about 8 orders of magnitude too high. In order to match the forces measured, there would need to be on the order of 30 charge groups per microsphere, which puts the surface density down to about one charge group per 200,000 square nanometers. There must be something neutralizing the surface charges down to this much lower density. The water in the experiment is de-ionized, so there should be very few charges $\left(10^{-7} \mathrm{~mol} / \mathrm{L}\right)$ available in the water to neutralize the charges. There might be some charges from the air as the sample is being made, and there might be some in the mineral oil, considering that the beads are hydrophobic and the majority of the bead sits in the oil when it is on the interface. Also, the beads could pick up neutralizing charges from each other. The dielectric constant of water would reduce the electric field of the beads by a factor of 80 , but again, only a portion of the bead is in the water.

Most of the data taken with the QPD is done at $1 \mathrm{kHz}$. For measurements that involve moving the beads toward each other, this is much faster than the timescale over which the forces change. The FSM is normally making small jumps on the order of 10-200 Hz. As the jump occurs, the bead will experience a drag force, but then it settles to a steady state force that it experiences from the other bead. It is possible to filter out noise and data that happens at timescales faster than the steady state forces being measured.

Quantization error occurs when dSpace measures the FSM voltage and QPD voltage. Instead of a continuum of voltages, the signals are so small that there are noticeable periodic gaps in the data. These can be eliminated by proper amplification. Already the signal from the QPD is amplified 10 times when it comes through the AA filter.

The optical tweezer does exert forces on the beads that must be taken into account when determining the potentials governing the behavior of the hexagonal lattice. There is a force between the trapped particles caused by the fact that they are both trapped and the light that is trapping them is scattered [30]. The force can be either attractive or repulsive depending on the distance between the two particles. This could explain the small oscillation in the force measurement. 


\subsection{SUMMARY}

It was found that a combination of the electrostatic Coulomb force and dipole-dipole interaction force dominated the pair interaction between the colloidal particles in the experiment. This is not surprising because experimental conditions were not close enough to ideal to measure any subtle, smaller interactions. The effective charge on the beads was found to be much lower than that given on the data sheet from the manufacturer. There are many factors that could have contributed to this. The lack of an absolutely clean lab set up could have introduced ions that could neutralize the sulfate groups on the microspheres.

It is interesting that the expected Coulomb forces due to the manufacturer's specified surface charges are orders of magnitude too great to even be measured with an optical trap. It must be common for the surface charges to be neutralized otherwise, the spacing in the lattice would be much larger.

A value is provided for the effective interfacial viscosity. The value is lower than that

of water or mineral oil, implying that surface forces somehow make movement along the interface easier than in bulk. 


\subsection{FUTURE WORK}

It would be useful to check the slope of the QPD voltage vs. beam/bead position for a few different particle sizes. This is because the slope of the linear part of the QPD response

should be proportional to $1 / d[15]$. Only one size of particle was used in this experiment, so this was not able to be checked.

It would be interesting to see the effect of different hydrophobicities on the measured interfacial viscosity. A relation could be found between the two interfacing fluid viscosities, and the effective viscosity of the interface. Also, it is important to find whether the beads are rolling on the interface, or whether they are pinned. This could be found by using a marked bead.

Over time it seemed that the strength of the electrostatic repulsion between the beads diminished. Several papers mentioned the cleaning of the beads to remove surfactants which could move from the bead to the interface. One specific process is the use of mixed-bed ion exchange resin. This would reduce the contact angle between the bead and water, causing increased shielding, and less interaction. In the future, it would be beneficial to use washed beads in the experiments to nullify the effect of the time the beads sit in solution before being used.

It was not possible to measure the curvature of the interface using the available equipment. With some way to move the focus knob of the microscope, and a motorized stage to move the well, it would be possible to measure the z-coordinate of the interface for the whole well. It is definitely not flat, but does not seem to simply follow the Young-Laplace equation either. 
The properties of the optical trap should be examined at both the center of the well where the curvature will be around zero, to the edges of the well where curvature is highest. Most of the future work will involve creating Bessel beam traps and exploring their unique properties and uses. The sections below will discuss this topic.

\subsection{BESSEL BEAMS}

A Bessel beam has its wavefronts converging on each other in a conical pattern. Bessel beams were first used in an experiment by Durnin, Miceli, and Eberly in 1986 [19]. Collimated light was sent through a circular slit placed in the focal plane of a lens. This created a beam of converging plane waves, such that the wavevector of every photon was parallel to the surface of a cone (its base being the slit)[19]. Bessel beams can be created by several methods (annular slit, axicon, or spatial light modulator) but in this work it is suggested to be formed by passing a collimated Gaussian beam through an axicon (conical lens). The Bessel beam has an 'extended' focus, that will allow trapping in several planes. Because the beads are adhered to the oil/water interface, 3D trapping is unnecessary, and force measurements can still be made.

The Rayleigh range of the focus of a Gaussian beam is very small. In this experiment, the $N A=0.75$ and $\lambda=1064 \mathrm{~nm}$. This gives a minimum waist size (radius) of the Gaussian beam:

$$
R=865.4 \mathrm{~nm}
$$




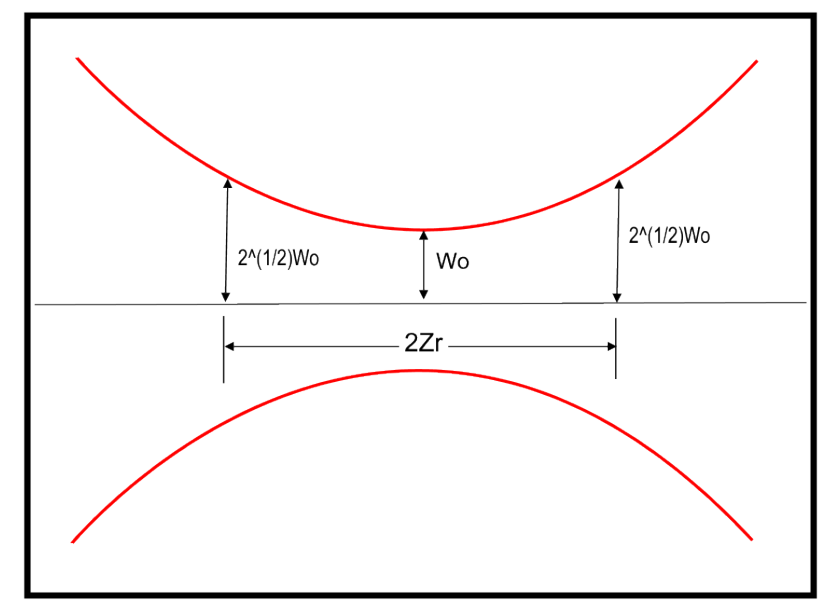

Figure 39: Gaussian beam characteristics.

Substituting this value into the equation for the Rayleigh range gives a "usable trapping tube length" of:

$$
\begin{gathered}
Z_{\text {Rayleigh }}=\frac{\pi r^{2}}{\lambda} \\
Z_{\text {Rayleigh }}=\frac{\pi\left(865.4 \cdot 10^{-9}\right)^{2}}{1064 \cdot 10^{-9}} \\
Z_{\text {Rayleigh }}=2.21 \mu \mathrm{m}
\end{gathered}
$$

which is barely greater than the diameter of one bead in the experiment.

Figure 39 shows the characteristics of the Gaussian beam. The distance $2 Z_{r}$ is the "usable trapping tube distance" which can be compared to the much longer distance of the Bessel beam (see Figure 40). The intensity at the focus drops off quickly and only that focal volume can be used for trapping. When force measurements are made, the object must be kept in the plane of the focus for the measurements to have a consistent meaning. If the trap does not confine the particle in three dimensions, there may be axial movement as a measurement is being made. If the particle is being pushed along on the optical axis as it is 


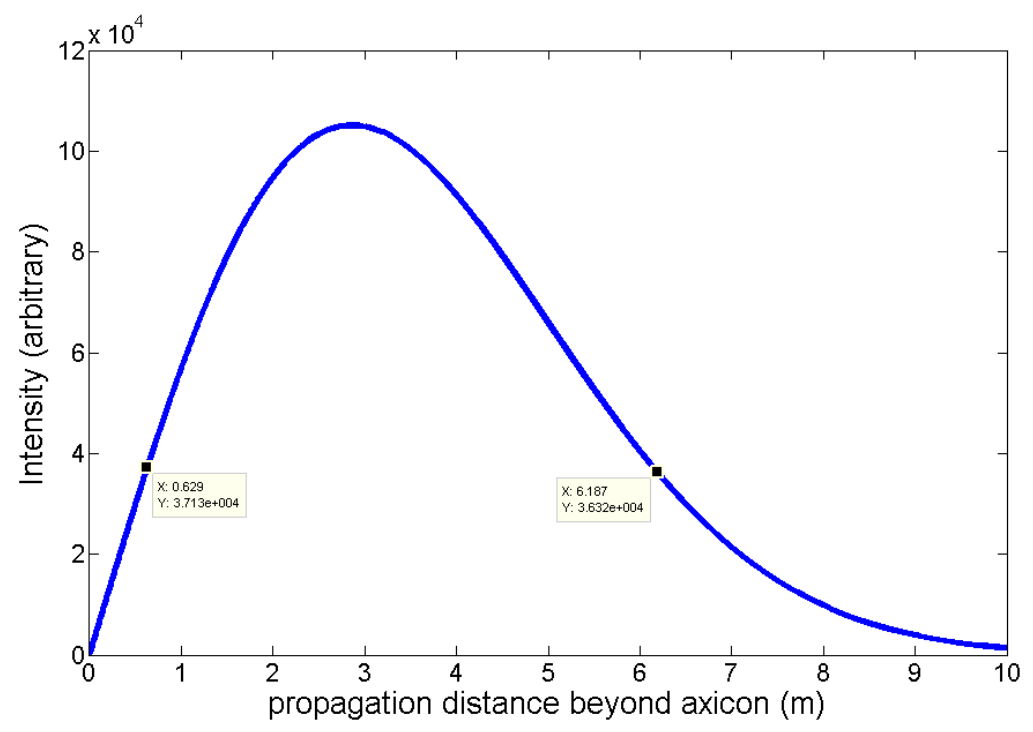

Figure 40: Two dimensional plot of Bessel beam along the optical axis.

being dragged through water, the drag force on the particle might not be consistent because different depths of the water might have different flow rates. It would be possible to measure the force between two objects that are not in the same focal plane if one beam was designed to be out of focus in the focal plane of the microscope, so that it is in focus at the depth of the object that needs to be trapped.

There is a characteristic radius of the beam out to the first intensity minimum, $r_{b}$, and a characteristic length that the beam propagates without diffracting, $z_{\max }$.

$$
\theta \approx\left(\frac{\pi}{2}-\frac{\alpha}{2}\right)\left(\frac{n_{a}}{n_{m}}-1\right)
$$

where $n_{a}$ is the refractive index of the axicon, $n_{m}$ is the refractive index of the propagating medium, $\alpha$ is the usually obtuse apex angle of the triangular cross-section of the cone of the axicon, and $\theta$ is the "polar angle between propagation axis of the Bessel beam and the plane waves wavevectors forming the Bessel beam behind the axicon" [21]. 
The propagation distance is:

$$
z_{\max }=w \frac{\cos \theta}{\sin \theta}
$$

where $w$ is the half-width of the incident beam at the axicon [21].

The radius of the central lobe of the Bessel beam is:

$$
r_{b}=\frac{2.4048}{k \sin \theta}
$$

where $k$ is the wavenumber of the beam in the medium [21]. For a reasonable $\theta$ :

$$
\begin{gathered}
\theta \approx\left(\frac{\pi}{2}-\frac{179 \text { degrees }}{2}\right)\left(\frac{1.52}{1.0}-1\right) \\
\theta \approx 0.004538 \text { radians } \\
z_{\text {max }}=\frac{0.0254 \text { meters } \frac{\cos 0.004538}{2}}{\sin 0.004538} \\
z_{\max }=2.8 \text { meters }
\end{gathered}
$$

Because the $z_{\max }$ scales with the square of the magnification, the $63 \mathrm{x}$ objective lens will cause the real $z_{\max }$ to be:

$$
\begin{gathered}
z_{\max }=\frac{2.8 \text { meters }}{63^{2}} \\
z_{\max }=13.5 \text { micrometers }
\end{gathered}
$$

which is about 6 times the "usable trap length" of the Gaussian trap.

Many industries and academic subjects require measurements to be made on forces acting in a direction other than perpendicular to the specimen plane. A Bessel beam can be used to extend the use of the optical trap to these situations. The equivalent $2 \mathrm{D}$ force between two particles that are out of plane would be the measured force divided by the cosine of the angle between them. Knowing that the Bessel beam has a uniform intensity over a long range can actually allow the mapping of the out of plane interface by the component of the force measured. For example, the optical tweezer created by a long working distance lens 

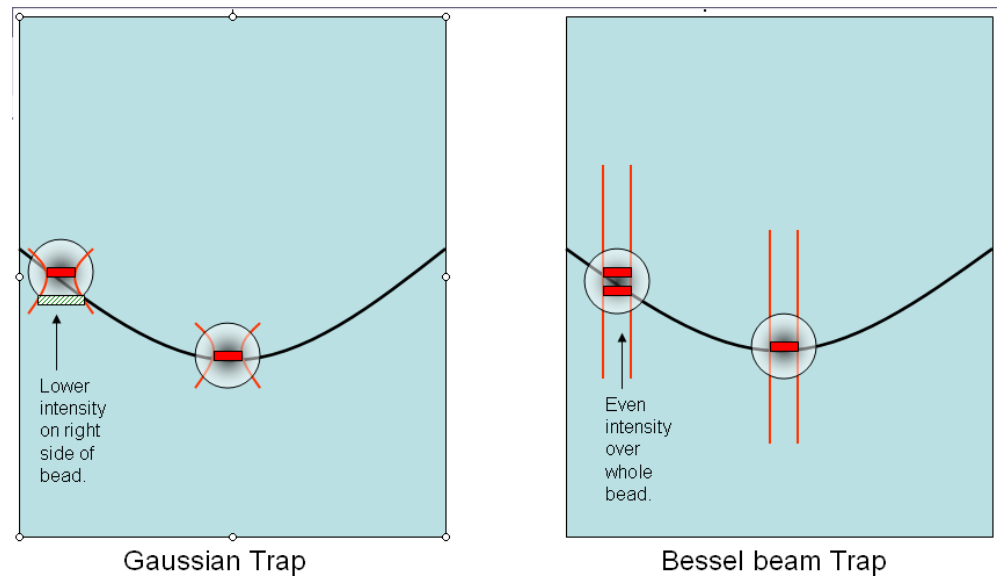

Figure 41: The use of a Bessel beam more accurately determines a force acting on a particle adsorbed to curved interface. This is because the intensity is the same at all points where the bead comes into contact with the interface.

only traps in two dimensions. If there is a third dimension to the interface, the component of the $2 \mathrm{D}$ force will be proportional to the local curvature of the interface. Figure 41 shows an illustration of this concept.

The interface of the beads has some curvature because the meniscus of the water creeps up the edges of the well. The Bessel beam trap should show much less variation between forces measured on the flat center of the interface and the steeply curved edges of the interface. Figure 41 shows an illustration of this concept. This assumes that the upward force of the Bessel beam trap is negligible on the curved interface. If it is not negligible, then the interface shape measuring will be enhanced. Dai et al. found that the ratio between the particle radius and the radius of curvature of an interface becomes less significant as the viscosity of the non-water phase becomes much greater than that of water ( $>10$ times)[28]. The viscosity of the mineral oil is about 30 times that of water. Therefore in this experiment, the drag caused by the curving of the interface should be negligible. 
A good way to compare the Gaussian trap with the Bessel beam trap would be to use a "volume trapping efficiency" which would look something like:

$$
q_{v o l}=\frac{\text { trap stiffness }}{P_{l a s e r}} \cdot t_{v o l}
$$

where $t_{v o l}$ is the volume over which the stiffness does not fall by a factor of e.

In order to fully use the capabilities of long-working distance optical trapping, it is interesting to investigate the uses of a Bessel beam which has a long "tube focus" which can reach deep into a sample and through a much longer region than a Gaussian focused beam.

There are two unique aspects to a Bessel beam, its long diffractionless propagation and its self-healing ability. Most experimental use of Bessel beams involves using them to guide particles, but not to measure forces.

The Bessel beam is a non-diffracting solution to the Helmholtz equation [19]. The Helmholtz equation:

$$
\left(\nabla^{2}+k^{2}\right) A(x, y, z)=0
$$

where $\nabla^{2}$ is the Laplacian and $\mathrm{k}$ is a constant. If $k=0$ then it reduces to the Laplace equation.

An axicon takes a point source and images it as a "tube" on the axis of the lens [29]. The axicon can be thought of as a toric lens in which the toroidal portion has an infinite radius [29].

J. Durnin, J. J. Miceli, and J.H. Eberly determined that Bessel beams are comparable to Gaussian beams for energy transport because they do not diffract, even though their peak intensity is lower [20].

A zeroth-order Bessel beam is created by an axicon. Zeroth order meaning that there is a central maximum. It is an approximation to a theoretical Bessel beam. A true Bessel beam would carry all its energy to infinity and is impossible to create. The beam within the propagation distance is what a true Bessel beam would look like extending to infinity. 
The equation describing how the Bessel beam propagates without decay/diffraction is $[20]:$

$$
\psi(x, y, z, t)=e^{\imath(\omega t-\beta z)} J_{0}(\alpha \rho)
$$

where $\psi$ is the wave amplitude, and it can be seen to oscillate, but not decay. Another description of the Bessel beam's electric field amplitude [18]:

$$
E(r, z)=A \exp \left(i k_{z} z\right) J_{0}\left(k_{r} r\right)
$$

where $k_{r}$ and $k_{z}$ are the radial and longitudinal parts of the wave vector, $\mathrm{k}$, respectively.

The reconstruction distance, i.e. the amount of distance it takes for a Bessel-beam to reform after an obstacle, is dependent on the refractive index of the obstructing particle[41]. This means Bessel beams could be used to find the refractive index of particles [41]. It should be noted that the beam will not reform if the whole beam is blocked by an opaque particle.

According to Garces-Chavez, et al, the amount of distance it takes for the Bessel beam to reform after an obstacle is[41]:

$$
l=\frac{r_{o b} k}{k_{r}}
$$

where $\mathrm{l}$ is the length of the shadow, $r_{o b}$ is the radius of the obstructing object, $\mathrm{k}$ is the wavevector and $k_{r}$ is the radial wavevector where

$$
k=\left(k_{r}^{2}+k_{z}^{2}\right)^{\frac{1}{2}}
$$

The reconstruction distance after the whole central spot is blocked is:

$$
d_{r} \approx \frac{3 a}{\tan \theta}
$$

where $d_{r}$ is the reconstruction distance, $a$ is the radius of the central spot and $\theta$ is the same as defined below [34]. 


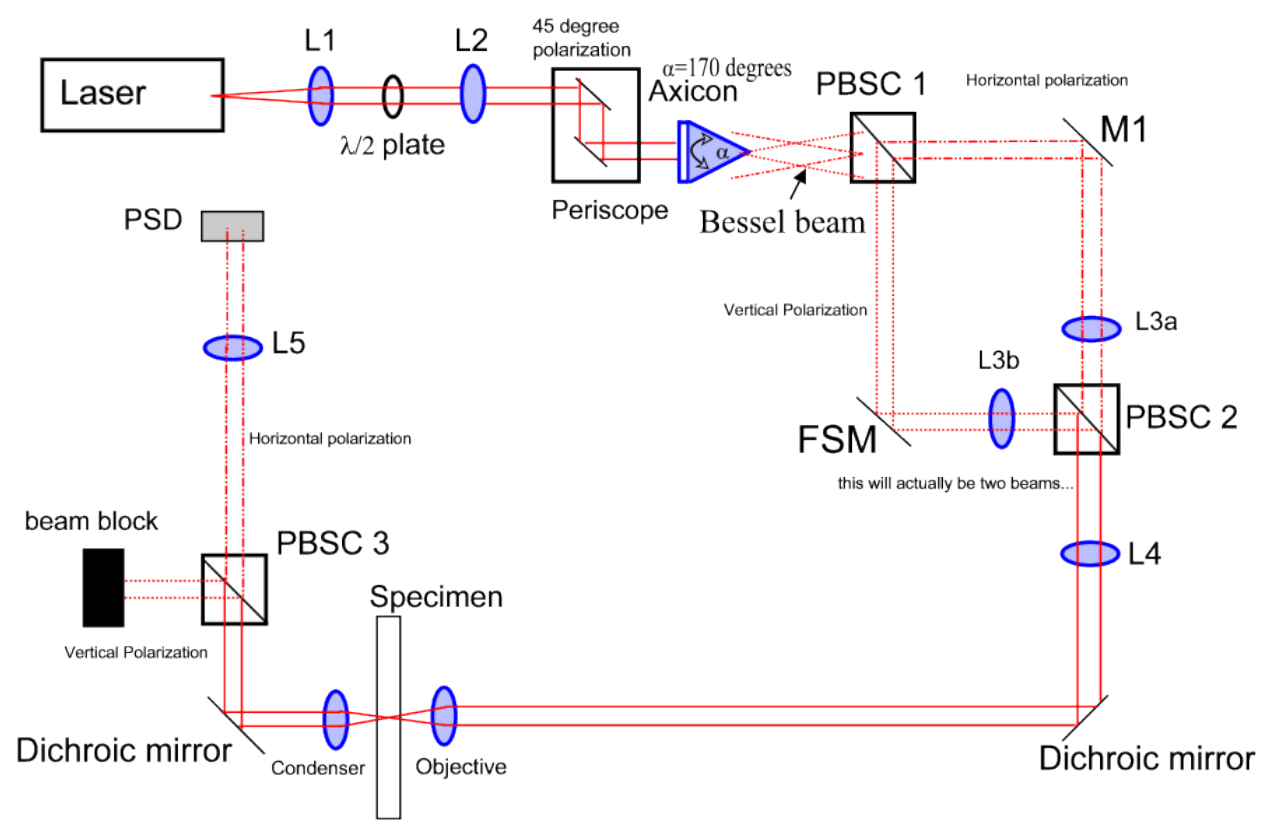

Figure 42: Optical Tweezer Setup with Axicon. 


\subsubsection{Choosing an Axicon}

The determination of what axicon to use in the experiment was made using equations found in Jezek et al. The schematic of the set-up for the "Bessel beam" portion of the experiment is shown in Figure 42. There is a characteristic radius of the beam out to the first intensity minimum, $r_{b}$, and a characteristic length that the beam propagates without diffracting, $z_{\max }$.

$$
\theta \approx\left(\frac{\pi}{2}-\frac{\alpha}{2}\right)\left(\frac{n_{a}}{n_{m}}-1\right)
$$

where $n_{a}$ is the refractive index of the axicon, $n_{m}$ is the refractive index of the propagating medium, $\alpha$ is the usually obtuse apex angle of the triangular cross-section of the cone of the axicon, and $\theta$ is the "polar angle between propagation axis of the Bessel beam and the plane waves wavevectors forming the Bessel beam behind the axicon" [21].

The propagation distance is:

$$
z_{\text {max }}=w \frac{\cos \theta}{\sin \theta}
$$

where $w$ is the half-width of the incident beam at the axicon [21].

The radius of the central lobe of the Bessel beam is:

$$
r_{b}=\frac{2.4048}{k \sin \theta}
$$

where $k$ is the wavenumber of the beam in the medium [21].

These values are for the Bessel beam that is produced immediately after the axicon. The lenses downstream affect the properties. Because the two telescopes in this experimental set-up (as shown in Figure 42 have a magnification of unity, they do not effect the properties of the Bessel beam. The 63x magnification of the long-working distance objective does affect the propagation distance and radius of the beam. Using the small angle approximation because the axicon has an apex angle of almost 180 degrees, the value of $r_{b}$ scales with the magnification and the value of $z_{\max }$ scales with the square of magnification [21]. 


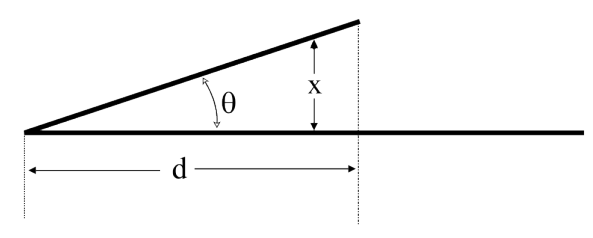

Figure 43: Variables in propagation matrix.

Ray transfer matrices for the action of propagation and a lens:

$$
\begin{aligned}
& {\left[\begin{array}{ll}
1 & d \\
0 & 1
\end{array}\right] \quad\left(\begin{array}{l}
x \\
\theta
\end{array}\right)=\left(\begin{array}{l}
x^{\prime} \\
\theta^{\prime}
\end{array}\right)} \\
& {\left[\begin{array}{cc}
1 & 0 \\
\frac{-1}{f} & 1
\end{array}\right] \quad\left(\begin{array}{l}
x \\
\theta
\end{array}\right)=\left(\begin{array}{l}
x^{\prime} \\
\theta^{\prime}
\end{array}\right)}
\end{aligned}
$$

The first equation is for propagation of a ray. The second is for a lens. Propagation changes only the $x$ value of the ray, while a lens changes only the angle.

In order to choose an axicon, assume a 1 inch diameter axicon because they are most readily available. Values for $\alpha$ (apex angle of axicon) range from 140 degrees to 179.9 degrees. The refractive index of the axicon, the refractive index of air, an average of the refractive indices of water and mineral oil, a Gaussian input beam of a half-width of $1 / 2$ inch are inputs and a variety of $\alpha$ values are tested. The propagation and lens matrices are used to trace the path of two rays, the center ray at the tip of the axicon, and the ray at the edge of the axicon. The maximum displacement from the optical axis determines the size of the lenses needed for the system. 
It was found that for $\alpha=170$ degrees, the edge ray is displaced a maximum of 1 inch off the optical axis, requiring a 2 inch lens. This lens is not the objective, and therefore can be made to be 2 inches in diameter. This set up provides a Bessel beam that extends 135 microns when the change in refractive index from air to water/oil is used. The radius of the zero order of the beam (i.e. the part of the Bessel beam to be used for trapping) is calculated to be 170 nanometers in the sample, but diffraction will cause this to be on the order of the wavelength of light (1 micron).

There are several apertures that the beam must be made to fit through. This makes the choice of axicon more difficult. The aperture of the objective is $6.5 \mathrm{~mm}$. There is an aperture before the first dichroic mirror that is 1 inch in diameter.

The Matlab script assumed the position and focal length of the two lenses closest to the specimen plane as shown in Figure 6.

The Bessel beam fully forms when it is half of $z_{\max }$ down the optical train. The equation of the plot uses the solution of the Fresnel integral by the stationary phase approximation method. A figure showing a two dimensional plot along the optical axis $(r=0)$ is shown in Figure 40. The point that are marked on the plot are the $1 / e$ points of the intensity defining the $z_{\max }$ range of the Bessel beam. The Fresnel integral follows the form [42]:

$$
\int_{a}^{b} e^{\frac{-i k x^{2}}{2 l}} d x=\left(\frac{2 \pi l}{k}\right)^{\frac{1}{2}} e^{\frac{-i \pi}{4}}=\sqrt{l \lambda} e^{\frac{-i \pi}{4}}
$$

where $l$ is the propagation distance, and $\lambda=\frac{2 \pi}{k}$.

The approximate solution is:

$$
I(r, z)=\frac{4 P k \sin \theta}{w} \frac{z}{z_{\max }} J_{0}^{2}(k r \sin \theta) e^{\left(\frac{-2 z^{2}}{z_{\max }^{2}}\right)}
$$

where $r$ is the radius from the optical axis [21]. This approximate equation is valid if [22]:

$$
z>\frac{z_{\max }^{2}}{k w_{0}^{2}}
$$

By the boundary condition of the input beam being a certain intensity, the intensity on the other side of the axicon should not be zero. This is an artefact of the approximation. 


\subsubsection{Preliminary Results and Observations}

During experimentation, it was noticed that the Bessel beam cannot be moved in an "aligned" way. The beam doesn't move as a perpendicular tube, in just the x-y plane of the specimen plane when it is controlled by the FSM. This may have to do with the fact that the axicon does not have a well-defined "focal length" and therefore it is not possible to place the FSM exactly in the back focal plane of the axicon. When moving the BB trap with the FSM, the trap not only translates, but the angle of the beam through the sample also changes. This is not desirable. In the literature review, it is rare that a particle is moved in a non-axial direction with the $\mathrm{BB}$. If this functionality is necessary, a motorized stage is used. Most times, the $\mathrm{BB}$ is stationary, or its properties can be moved axially by moving the axicon parallel to the beam or changing the SLM hologram. This is very important with regards to the usefulness of a BB. The only paper in the literature review where the Bessel beam

was not stationary was by J. Arlt, et al in 2001. In it, the position of the Bessel beam is controlled by moving the lens after the axicon in 3 dimensions but NO force measurements are made [18].

A paper in which a force measurement is made with a Bessel beam was not found during the literature review. This could be because the beam is self-healing, making this impossible without a coincident beam.

\subsection{BESSEL BEAM TRAP PAIR POTENTIAL MEASUREMENTS}

Try to keep as many parameters constant as possible, i.e. laser power. Because the Bessel beam is self-healing, the light that is scattered from the bead cannot be used to gather information about the position of the bead. No matter how the bead obstructs or alters the path of the light, the signal at the QPD remains the same. In order to measure the force exerted by a Bessel beam, it is necessary to coalign another "measurement beam" that is too weak to trap, but intense enough to shine through the particle and provide information about its position. This set-up seems to only allow pair potential measurements because 
the FSM beam will be a Gaussian trap and the stationary beam will contain the Bessel beam and the red "measurement beam". Drag measurements through bulk water will not be possible with this method, so only power spectral density measurements will provide the stiffness of the Bessel beam trap at a certain power. The force between two particles at a certain distance is known from the Gaussian trap data, therefore the measurement with the Bessel beam will be only a formality to confirm that the same force is measured with both the Gaussian and Bessel beam traps according to their respective stiffnesses.

The beads just below and just above the interface may be trapped by the Bessel beam. This should still not be a problem because the "measurement beam" is Gaussian and will only scatter light from the bead trapped on the interface. The only problem would come from beads being pushed up into the interface while the measurement is being taken. This same problem occurs for regular Gaussian trap pair potential measurements.

The axicon chosen for the experiment has an $\alpha=170$ degrees.

$$
\begin{gathered}
\theta \approx 0.045 \\
z_{\text {max }}=0.01 m \frac{\cos 0.045}{\sin 0.045} \\
z_{\text {max }}=0.222 \mathrm{~m}
\end{gathered}
$$

The radius of the central lobe of the Bessel beam is:

$$
r_{b}=\frac{2.4048}{k \sin \theta}
$$

where $k=\frac{2 \pi}{\lambda}$ but maybe account for different refractive indices, so possibly off by factor of 1.33 or 1.5 .

$$
\begin{aligned}
r_{b} & =\frac{2.4048}{\frac{2 \pi}{1064 \mathrm{~nm}} \sin 0.045} \\
r_{b} & =9 \text { micrometers }
\end{aligned}
$$

without any magnification. 


\section{APPENDIX}

\section{WHOLE PROCEDURE}

1. Set up optical trap.

2. Check alignment.

a. Focus on thumbprint and make sure everything looks right.

b. Adjust condenser.

c. Make sure beam is collimated at QPD.

d. Choose a laser power to use for all measurements.

3. Dry beads onto a coverslip.

a. Mark the inside of the coverslip and the slide to have markings with which to compare the actual location of the beads.

b. Seal to slide with water.

c. Seal to slide with oil.

d. Repeat with drying beads to the slide. It seems that the contrast (difference between min and max reading) is best with beads dried on the slide, not the coverslip.

e. Perform all measurements at two power levels. One level will be designated "LOW" and will be used to raster scan the beads when they are trapped by the stationary beam. The other power level will be designated "TRAP" and will be the power of the beam during FSM trapping.

f. This type of measurement (raster of stationary bead) will enable the determination of how far a bead is pushed out of the center of the beam by an external force. 
Specifically using dried-on beads is only necessary as a first-time sanity check to make sure the QPD is functioning properly. This data can also be used to qualitatively figure out the effects of different refractive indices on light scattering and QPD data. Sometimes there were different blocks hooked to the input of the FSM. For example, a raster would involve a "repeating sequence stair".

g. NOTE: This dried-on bead step does not need to be repeated for every experimental set-up change.

h. A Matlab model of the scattering for the four cases gives a maximum difference of 2 percent. This does not match with the measured data shown in Table 4. Possible explanations are that the oil absorbs more light than water, or that the spherical nature of the particles has a large effect on the scattering, and the slab approximation is not appropriate.

i. The QPD voltages also depend on where the beam is focused in the $\mathrm{Z}$ direction, into the sample. As the focus is moved from the shallowest region of the bead, through the bead to the deepest, the bead apparently grows and shifts according to the QPD voltages. This means that the measurements taken later in the experiment will also depend on focus depth. Because the trap is not focused tightly enough, the beads get pushed in the $\mathrm{Z}$ direction by the trap, so the bead will not self-center itself in the trap. The focus depth will matter.

j. The data shows that the dynamic range of the QPD measurement, i.e. the difference between the maximum and minimum voltage on any particular scan is proportional to the laser power. This means that data taken at "LOW" power in bulk raster scans can be extrapolated to correlate with data taken at "TRAP" power.

4. Take FSM volts per nanometer measurement:

a. with dried bead/water slide.

b. with dried bead/oil slide.

- Take care to focus on beads that are on the cover slip and not the slide. BE CONSISTENT.

- There is no difference in Volts/nm of the FSM whether the microscope is focused on the coverslip surface or the slide surface. 
- Use the "VperNMcal.mdl" file in Calibration/calibration in matlab to get a 2D cross section of the beads. The raster can be done with "fourinputcal".

- When taking measurements with the QPD, zero it by checking what position will give a "no-beam" response. This is not zero volts. It is more like $-1.1 \mathrm{~V}$ for one channel and $-1.6 \mathrm{~V}$ for the other channel.

c. The vectors for the repeating sequence stairs in Simulink can be created using "beamvector.m".

d. The Simulink model is "fourinputcal" in the same folder. The sequence stairs can be replaced with constants to control the location of the beam by hand in dSpace.

e. This measurement will enable the determination of velocity that the bead is dragged during calibration experiments.

f. It is expected that the FSM Volts per pixel moved across the microscope screen should be the same for all cases from beads dried on slides, to beads trapped in bulk, to beads trapped at an interface. Measurements have determined a maximum deviation for the Gaussian trap case. Table 3 shows the relationship between FSM volts and pixels the beam moves across the screen in the horizontal and vertical directions for several cases of the Gaussian trapping set-up. The thickness of water that the beam travels through in the "bulk water" case is approximately 100 microns while in the "interface" case, the beam travels through about 500 microns of water. The fact that both cases are within 5 percent of each other implies that the beam is going straight through the sample at all FSM voltages tested. There are only small angles introduced to the beam.

g. Sanity Check - The signals of QPDx and FSMx should have high coherence and the signals of QPDy and FSMy should have high coherence. This can be measured by finding the transfer function between the FSM input and the QPD output. It is important that in a scan of the bead, that the beam is only scanned within the linear portion of the QPD's response curve. This means the beam should stay within about a half micron from the center of the bead. Non-linearities show up as extra noise. Figure 19 shows the system that was used to make the coherence measurements etc. h. In the linear region of the QPD response, the FSM to QPD transfer function should 
be flat. It can be seen in Figure 19 that one AA filter applies a gain of one to the signal coming from the dSpace board while the other AA filter applies a gain of 10 to the signal coming from the QPD to increase the dynamic range of measurement. The trailing off of the data around $400 \mathrm{~Hz}$ in Figures 20 and 21 is due to the 500 $\mathrm{Hz}$ cutoff of the AA filter. The fact that the magnitude of the gain is not one may be due to the fact that there are factors of 10 between the input to dSpace and the output for which may not have been accounted. The non-zero slope of the phase shows that the "group delay" of this set-up is not constant. This means that there is dispersion (certain frequencies are delayed more than others).

i. The coherence of an input/output system has to do with how related the output is to the input. A coherence of one says that a certain input after a certain initial condition produces the same response every time at the output. It is desirable for this set-up to have a coherence of one in all four cases, FSMy to QPDy, etc. It is shown in Figures 22 and 23 that the coherence is near one for all pertinent frequencies in the two corresponding channels (FSMy to QPDy and FSMx to QPDx). the cross channels are not as important, but they are also around one for most of the spectrum.

5. Prepare a slide with beads in bulk:
a. water.
b. oil.

6. Trap a bead in bulk and measure the frequency response at $1 \mathrm{kHz}$, with AA filter at 3 $\mathrm{kHz}$.

a. water

b. oil

c. The trap will be assumed stationary here, so the data collected at the QPD can be used to determine the distance the bead is pushed out of the trap by Brownian motion.

7. Determine stiffness from equipartition theorem using 'stiffnesscalnew' in Calibration/ calibration in Matlab.

8. Trap bead in bulk and drag through square at 3 different velocities 
a. water.

i. It is necessary to save the vectors which define where the bead is dragged because these will be used to determine the actual velocity at which the bead is dragged.

b. Take a measurement with no bead and subtract as an offset.

i. The no bead measurement needs to be taken at every velocity at which data is collected for it to be possible to subtract the voltages.

9. Determine stiffness of trap from force vs. velocity with 'forcevleplotbad.m' in Calibration/calibration in Matlab.

a. Also drag the beads back and forth in $1 \mathrm{D}$ for both $\mathrm{x}$ and $\mathrm{y}$ with a sine wave. This will move the bead at many different velocities, and it will be possible to filter the data at that frequency to pick up "noiseless" information.

10. Prepare beads on interface.

a. A piece of $1.53 \mathrm{~mm}$ thick plastic with a $5 \mathrm{~mm}$ hole drilled in it, is glued with nail polish and superglue onto a No. 1 coverslip.

b. A microsyringe is used to place about 15 microliters of deionized water into the well.

c. A $2 \mathrm{~mm}$ by $3 \mathrm{~cm}$ piece of aluminum can is dipped into a vial of mineral oil and the oil collects at the tip and is dropped into the well on top of the water.

d. The solution of microspheres, water, and isopropanol must be shaken very hard to make sure the water and alcohol is mixed well.

i. Note: When the vial of microsphere solution is not shaken, beads will adsorb to the interface, but the hexagonal lattice will be less pronounced or not present at all, and measurements confirm that the beads do not exert the same force on each other in this state even though they still appear to be in a plane, i.e. on the interface.

e. Use the microsyringe to inject about 2 microliters of microsphere solution into the interface.

i. This can be done by hand.

ii. Start the tip of the syringe in the water and pull up through the interface and oil as the plunger is depressed. 
iii. The oil should react in such a way that it retreats from the injection site, ripples a little, and then re-covers the open patch of water that was created when it retreated. If this does not happen, it is likely that no beads were deposited on the interface.

11. Change ratio of polarizations and make the stationary trap much stronger than the FSM trap. (maybe stat trap is $300 \mathrm{~mW}$ and FSM trap is $80 \mathrm{~mW}$ ) The force on the bead is proportional to the power. Hold the bead still with the stationary trap and raster the beam across the bead. Hopefully it will not move appreciably and the measurement can be compared to the dried bead oil and water rasters.

12. The interface can have flow patterns. From the measurements taken, there was no noticeable pattern as to what conditions caused what flow patterns. These flow patterns increase in velocity and change direction depending on whether the laser is on, and which power setting is chosen. This can make trapping two beads to move towards each other very difficult. Even if two beads can be trapped, the flow causes other beads to bump into them disturbing the measurement being taken.

13. Drag bead slowly towards and away from another bead trapped with the stationary mirror in the shape of a cross. This will provide data on the force vs. position for four directions, i.e. from the right, left, top and bottom.

14. Take frequency response of bead on interface at $1 \mathrm{kHz}$.

15. Repeat steps 1-14 with axicon set up. Try to keep as many parameters constant as possible, i.e. laser power. 


\section{BIBLIOGRAPHY}

[1] J.E. Bjorkholm A. Ashkin, J.M. Dziedzic and Steven Chu. Observation of a single-beam gradient force optical trap for dielectric particles. Optics Letters, Vol. 11, No. 5:288-290, 1986.

[2] et al. Alexis I. Bishop. Optical microrheology using rotating laser-trapped particles. Physical Review Letters, Volume 92, Number 19:198104-1-198104-4, 2004.

[3] A. K. Arora and Raj Rajagopalan. Ordering and Phase Transitions in Charged Colloids. VCH Publishers, Inc., 1996.

[4] A. Ashkin. Acceleration and trapping of particles by radiation pressure. Physical Review Letters, 24:156-159, 1970.

[5] A. Ashkin. Forces of a single beam gradient laser trap on a dielectric sphere in the ray optics regime. Biophysics Journal, 61:569-582, 1992.

[6] A. Ashkin and Dziedzic. Observation of resonances in the radiation pressure on dielectric spheres. Physical Review Letters, 38:13511354, 1977.

[7] A. Ashkin and J. M. Dziedzic. Feedback stabilization of optically levitated particles. Applied Physics Letters, 30:202-204, 1977.

[8] Bernard P. Binks and Tommy S. Horozov, editors. Colloidal Particles at Interfaces. Cambridge University Press, 2006.

[9] et al Bum Jun Park. Direct measurement of the effects of salt and surfactant on interaction forces between colloidal particles at water-oil interfaces. Langmuir, 24:1686-1694, 2008.

[10] R. S. Burdon. Surface Tension and the Spreading of Liquids. Cambridge University Press, 1940.

[11] John C. Crocker and David G. Grier. Microscopic measurement of the pair interaction potential of charge-stabilized colloid. Physical Review Letters, Volume 73, No. 2:352355, 1994. 
[12] John C. Crocker and David G. Grier. Methods of digital video microscopy for colloidal studies. Journal of Colloid and Interface Science, 179:298-310, 1996.

[13] John C. Crocker and David G. Grier. When like charges attract: The effects of geometrical confinement on long-range colloidal interactions. Physical Review Letters, 77(9):1897-1900, 1996.

[14] F. Ghezzi and J. C. Earnshaw. Formation of meso-structures in colloidal monolayers. J. Phys.: Condens. Matter, 9:L517-L523, 1997.

[15] Frederick Gittes and Christoph F. Schmidt. Interference model for back-focal-plane displacement detection in optical tweezers. Optics Letters, 23, no.1:7-9, 1998.

[16] David G. Grier. A revolution in optical manipulation. Nature, 424:810-816, 2003.

[17] Alan J. Hurd. The electrostatic interaction between interfacial colloidal particles. J. Phys. A: Math. Gen., 18:L1055-L1060, 1985.

[18] W. Sibbett K. Dholakia J. Arlt, V. Garces-Chavez. Optical manipulation using a bessel light beam. Optics Communications, 197:239-245, 2001.

[19] J.J. Miceli J. Durnin and J. H. Eberly. Diffraction-free beams. Physical Review Letters, 58:1499-1501, 1987.

[20] Jr. J. Durnin, J.J. Miceli and J.H. Eberly. Comparison of bessel and gaussian beams. Optics Letters, 13:79-80, 1988.

[21] Vilem Nedela Jan Jezek, Tomas Cizmar and Pavel Zemanek. Formation of long and thin polymer fiber using nondiffracting beam. Optics Express, Vol. 14, No. 19:8506-8515, 2006.

[22] Josh Soneson Jochen Arlt, Kishan Dholakia and Ewan M. Wright. Optical dipole traps and atomic waveguides based on bessel light beams. Physical Review A, Vol. 63:0636021-063602-8, 2001.

[23] M.D. Jurgen R. Meyer-Arendt. Introduction to Classical and Modern Optics - 4th Edition. Pretice Hall, 1995.

[24] R. K. Kalia and P. V. Vashishta. Interfacial colloidal crystals and melting transition. J. Phys. C: Solid State Phy., 14:L643-L648, 1981.

[25] Shinji Kamimura and Ritsu Kamiya. High-frequency vibration in flagellar axonemes with amplitudes reflecting the size of tubulin. Journal of Cell Biology, Vol. 116, No. 6:1443-1454, 1992.

[26] Milton Kerker. The Scattering of Light - and other electromagnetic radiation. Academic Press, 1969. 
[27] Hyungchul Kim and Diane J. Burgess. Prediction of interfacial tension between oil and mixtures and water. Journal of Colloid and Interface Science, 241:509-513, 2001.

[28] Chih-Yuan Wu Shashidhar Guttula Lenore L. Dai, Sowmitri Tarimala and Jian Wu. The structure and dynamics of microparticles at pickering emulsion interfaces. Scanning, 30:87-95, 2008.

[29] John H. McLeod. The axicon: A new type of optical element. Journal of the Optical Society of America, Vol. 44, No. 8:592-597, 1954.

[30] Jean-Marc Fournier Michael M. Burns and Jene A. Golovchenko. Optical binding. Physical Review Letters, Volume 63, No. 12:1233-1236, 1989.

[31] V. Adrian Parsegian. Van der Waals Forces: A Handbook for Biologists, Chemists, Engineers, and Physicists. Cambridge University Press, 2006.

[32] Pawel Pieranski. Two-dimensional interfacial colloidal crystals. Physical Review Letters, Vol. 45, No. 7:569-572, 1980.

[33] et al. R. Aveyard, B.P. Binks. Measurement of long-range repulsive forces between charged particles at an oil-water interface. Physical Review Letters, Vol. 88, No. $24: 246102,2002$.

[34] T. Okamoto J. Chrostowski B. A. Syrett R. P. MacDonald, S. A. Boothroyd. Interboard optical data distribution by bessel beam shadowing. Optics Communications, 122:169$177,1996$.

[35] Tommy S. Horozov Robert Aveyard, John H. Clint. Aspects of the stabilsation of emulsions by solds: Effects of line tension and monolayer curvature energy. Physical Chemistry Chemical Physics, 5:2398, 2003.

[36] Steven Chu Robert M. Simmons, Jeffrey T. Finer and James A. Spudich. Quantitative measurements of force and displacement using an optical trap. Biophysics Journal, 70:1813-1822, 1996.

[37] D. J. Robinson and J. C. Earnshaw. Initiation of aggregation in colloidal particle monolayers. Langmuir, 9:1436-1438, 1993.

[38] Manuel Sickert and Francis Rondelez. Shear viscosity of langmuir monolayers in the low-density limit. Physical Review Letters, Vol. 90, No. 12:126104-1-126104-4, 2003.

[39] David F. Blair Steven M. Block and Howard C. Berg. Comliance of bacterial flagella measured with optical tweezers. Nature, 338:514-518, 1989.

[40] Paula Moldenaers Sven Reynaert and Jan Vermant. Control over colloidal aggregation in monolayers of latex particles at the oil-water interface. Langmuir, 22:4936-4945, 2006. 
[41] H. Melville W. Sibbett V. Garces-Chavez, D. McGloin and K. Dholakia. Simultaneous micromanipulation in multiple planes using a self-reconstructing light beam. Nature, 419:145-147, 2002.

[42] H.C. Van de Hulst. Light Scattering by Small Particles. John Wiley \& Sons, Inc., 1957.

[43] Koen Visscher and Steven M. Block. Versatile optical traps with feedback control. Methods in Enzymology, 298:460-489, 1998.

[44] John Y. Walz. Ray optics calculation of the radiation forces exerted on a dielectric sphere in an evanescent field. Applied Optics, Vol. 38, No. 25:5319-5330, 1999.

[45] Kurt D. Wulff. Adaptive Control of an Optical Trap for Single Molecule and Molecular Motor Research. PhD thesis, Duke University, 2007.

[46] Shifa Wu Xin Huo, Shi Pan and Wei Sun. Development of far field and near field optical trapping. Chinese Optics Letters, Vol. 3, Supplement:S71-S73, 2005. 